\title{
Viscous profiles in models of collective movement with negative diffusivity
}

\author{
Andrea Corli(i) and Luisa Malaguti
}

\begin{abstract}
In this paper, we consider an advection-diffusion equation, in one space dimension, whose diffusivity can be negative. Such equations arise in particular in the modeling of vehicular traffic flows or crowds dynamics, where a negative diffusivity simulates aggregation phenomena. We focus on traveling-wave solutions that connect two states whose diffusivity has different signs; under some geometric conditions, we prove the existence, uniqueness (in a suitable class of solutions avoiding plateaus) and sharpness of the corresponding profiles. Such results are then extended to the case of end states where the diffusivity is positive, but it becomes negative in some interval between them. Also the vanishing viscosity limit is considered. At last, we provide and discuss several examples of diffusivities that change sign and show that our conditions are satisfied for a large class of them in correspondence of real data.
\end{abstract}

Mathematics Subject Classification. 35K65, 35C07, 35K55, 35K57.

Keywords. Degenerate parabolic equations, Negative diffusivity, Traveling-wave solutions, Collective movements.

\section{Introduction}

We are interested in the advection-diffusion equation

$$
\rho_{t}+f(\rho)_{x}=\left(D(\rho) \rho_{x}\right)_{x}, \quad t \geq 0, x \in \mathbb{R} .
$$

The variable $\rho$ is understood as a density or concentration and is valued in the interval $[0,1]$; the flux $f$ and the diffusivity $D$ are smooth functions. The main assumption of our study is that $D$ changes sign, even more than once. Equation (1.1) is then a forward-backward parabolic equation. In spite of the fact that these equations are well known to be unstable in the backward regime, nevertheless they arise in a natural way in several physical $[12,24]$ and biological [22] models.

Equation (1.1) also arises in the modeling of collective movements, for instance, vehicular traffic flows or crowds dynamics. In these cases, vehicles or pedestrians are assumed to move along a straight road or corridor, respectively; their normalized density at time $t$ and place $x$ is represented by $\rho(x, t)$. The corresponding flow is $f(\rho)=\rho v(\rho)$, where the velocity $v$ is an assigned function. The first models proposed in $[28,36]$ had no diffusivity; we refer to $[15,37]$ for updated information on this case. Then, the densityflow pairs lie on a curve (the graph of $f$ ) in the $(\rho, f)$-plane. However, experimental data show that this is not the case $[18,23]$ : such pairs usually cover a two-dimensional region. To reproduce this effect, either one considers second-order models $[1,34,45]$ or, as in this paper, introduces a diffusive term. In the latter case, the physical flow is $q=f(\rho)-D(\rho) \rho_{x}$, see $[4,5,7,32]$, and the density-flow pairs now correctly cover a full two-dimensional region in the $(\rho, q)$-plane. Moreover, the introduction of $D$ avoids the appearance of shock waves and then the occurrence of an infinite acceleration, which do not seem to fit well with the usual perception of collective flows. We refer to $[4,5,7]$ for several models where the diffusivity $D$ can vanish, but otherwise remains positive. The negativity of $D$ simulates an aggregative behavior; it occurs, for instance, in vehicular flows for high car densities and limited sight distance ahead [32]. An analogous 
modeling can be made in the framework of crowds dynamics; in this case, as we propose in this paper, it may simulates panic behaviors in overcrowded environments [9].

In the case $f=0$, a general framework to Eq. (1.1) has been proposed in $[13,35]$ to cope with the instability of solutions and the loss of uniqueness [21], even in several space dimensions. The "correct" solutions to the equation (with or without boundary conditions)

$$
u_{t}=\Phi(u)_{x x}
$$

are characterized as limits for $\varepsilon \rightarrow 0$ of the solutions of the pseudo-parabolic approximation

$$
u_{t}^{\varepsilon}=\Phi\left(u^{\varepsilon}\right)_{x x}+\varepsilon \Psi\left(u^{\varepsilon}\right)_{x x t} .
$$

Here, $\Phi$ is a nonmonotone function while $\Psi$ satisfies suitable assumptions of sign and growth. Solutions of (1.2) are understood in the sense of Young measures; they inherit some properties by the solutions of (1.3), which are dubbed entropy conditions for analogy with the hyperbolic setting. This approach has been studied in detail in several papers, see, for instance, [31] for initial data avoiding the unstable region, $[38,39]$ for the case when $\Psi^{\prime}(u) \rightarrow 0$ when $u \rightarrow \infty,[40,41]$ for initial data also valued in the unstable region. The drawback of this approach is that it is limited, for the moment, to the case $f=0$; moreover, the third-order term in the approximation (1.3) has no clear meaning for collective movements.

In this paper, we are concerned with solutions $\rho$ of Eq. (1.1) having low density in the past and higher density in the future, or conversely. To this aim, we restrict our investigation to traveling-wave solutions $\rho(x, t)=\varphi(x-c t)$. The profile $\varphi$ satisfies the differential equation

$$
\left(D(\varphi) \varphi^{\prime}\right)^{\prime}+(c \varphi-f(\varphi))^{\prime}=0,
$$

where the constant $c$ denotes its speed; more precisely, solutions are meant in the weak sense according to the following Definition 2.1. We are interested, in particular, in monotonic profiles defined in $\mathbb{R}$ and connecting a value $\ell^{-}$to a value $\ell^{+}$with $\ell^{-} \neq \ell^{+}$, i.e., such that

$$
\varphi(-\infty)=\ell^{-}, \quad \varphi(+\infty)=\ell^{+} .
$$

Moreover, we are concerned with the case when either $D\left(\ell^{-}\right)>0$ and $D\left(\ell^{+}\right)<0$, or $D\left(\ell^{ \pm}\right)>0$ but then $D$ is negative in an interval contained in $\left(\ell^{-}, \ell^{+}\right)$. As far as wavefronts are concerned, the special case when $f=0$ but Eq. (1.1) is endowed of a source term $g$ has been considered by many authors; we refer to $[2,3,29]$ for $D$ changing sign once and monostable $g$, [30] for the bistable case, $[14,26]$ for $D$ changing sign twice where $g$ is, respectively, monostable and bistable.

We refer to $[32,33]$ for several interpretations of the wavefront solutions to Eq. (1.1) in the case $D(\rho)=-\rho v^{\prime}(\rho)\left(\delta+\tau \rho v^{\prime}(\rho)\right)$ and in the framework of vehicular flows. Here, $\delta$ is an anticipation distance and $\tau$ a reaction time. However, even if also the case when $D<0$ is commented in [32], in [33] only the case when $D$ is positive is treated.

A special mention concerns the traveling-wave solutions constructed in [12] for a model of infiltration through porous media. In that case, the region where $D$ is negative is bypassed by inserting in the solution a shock wave, which is uniquely determined by a higher-order regularization (either of pseudo-parabolic type, as in (1.3), or of Cahn-Hilliard type); in this framework, the idea goes back to [44]. On the contrary, our (smooth) profiles shall be proved to travel also in the region of negative diffusivity, in spite of the fact the backward heat equation is well known to be highly unstable [22].

About Eq. (1.1), the existence of wavefront solutions in intervals where $D$ does not change sign follows by a result in [16]; this topic is briefly discussed in Sect. 4, where we extend that result to cover the case when $D$ is negative. On the other hand, the case when a wavefront solution crosses an interval where $D$ changes sign is more delicate and has been considered only in [17] for the case $f=0$ and $u(x, t)=\varphi(x / \sqrt{t})$. Our main results are provided in Sect. 2 and give necessary and sufficient conditions for the existence of wavefronts in the cases $D$ changes sign once or twice. We also study the smoothness of the profiles, in particular at the singular points where $D$ changes sign. At last, we prove the vanishing viscosity limit 


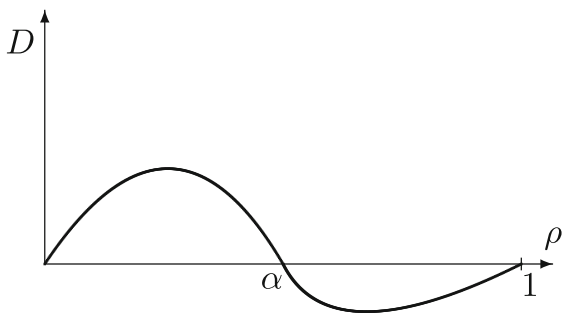

(a)

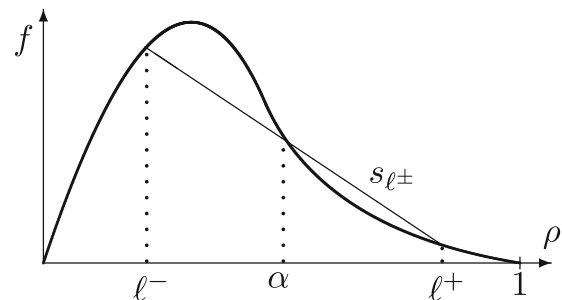

(b)

FIG. 1. a A diffusivity $D$ satisfying assumption (D1); b The flux function $f$

of the wavefronts to discontinuous (nonentropic) solutions to the corresponding hyperbolic conservation law

$$
\rho_{t}+f(\rho)_{x}=0 .
$$

As a consequence, Theorem 2.2 below shows that some nonclassical shock waves [27] considered in [9] (in the hyperbolic regime $D=0$ ), in the modeling of panic situation in crowds dynamics, admit a viscous profile. This topic is also discussed in Sect. 2. The proofs of our results are given in Sect. 5. The main applications are collected in Sect. 3; further examples are provided in Sect. 4.

\section{Main results}

We assume that the flux function $f$ and the diffusivity $D$ satisfy, for some $\alpha \in(0,1)$,

(f) $f \in C^{1}[0,1], f(0)=0$;

(D1) $D \in C^{1}[0,1], D(\rho)>0$ for $\rho \in(0, \alpha)$ and $D(\rho)<0$ for $\rho \in(\alpha, 1)$.

We notice that in this case we clearly have $D(\alpha)=0$; moreover, the condition $f(0)=0$ in (f) is not really an assumption because $f$ is defined up to an additive constant. We warn the reader that in Fig. 1 and in the following ones we represent $f$ with $f(\rho)>0$ in $(0,1), f(1)=0$ and $D$ with $D(0)=D(1)=0$. Such assumptions are common in dealing with collective movements, see Sect. 3, but are by no means necessary for the results below.

We now recall some definitions on traveling-wave solutions [16].

Definition 2.1. Let $I \subseteq \mathbb{R}$ be an open interval, $D \in C[0,1], f \in C[0,1] \cap C^{1}(0,1), f(0)=0$. Consider a function $\varphi: I \rightarrow[0,1]$ such that $\varphi \in C(I)$ and $D(\varphi) \varphi^{\prime} \in L_{\text {loc }}^{1}(I)$; fix $c \in \mathbb{R}$.

For all $(x, t)$ with $x-c t \in I$, the function $\rho(x, t)=\varphi(x-c t)$ is said to be a traveling-wave solution to Eq. (1.1) with wave speed $c$ and wave profile $\varphi$ if for every $\psi \in C_{0}^{\infty}(I)$

$$
\int_{I}\left(D(\varphi(\xi)) \varphi^{\prime}(\xi)-f(\varphi(\xi))+c \varphi(\xi)\right) \psi^{\prime}(\xi) \mathrm{d} \xi=0 .
$$

A traveling-wave solution is global if $I=\mathbb{R}$ and strict if $I \neq \mathbb{R}$ and $\varphi$ is not extendible to $\mathbb{R}$. It is classical if $\varphi$ is differentiable, $D(\varphi) \varphi^{\prime}$ is absolutely continuous and (1.4) holds a.e.; at last, it is sharp at $\ell$ if there exists $\xi_{\ell} \in I$ such that $\varphi\left(\xi_{\ell}\right)=\ell$, with $\varphi$ classical in $I \backslash\left\{\xi_{\ell}\right\}$ and not differentiable at $\xi_{\ell}$.

A global, bounded traveling-wave solution with a monotonic, nonconstant profile $\varphi$ satisfying (1.5) with $\ell^{-}, \ell^{+} \in[0,1]$ is said to be a wavefront solution from $\ell^{-}$to $\ell^{+}$.

Above, monotonic means that $\xi_{1}<\xi_{2}$ implies $\varphi\left(\xi_{1}\right) \leq \varphi\left(\xi_{2}\right)$. We denote by $s_{\ell^{ \pm}}=s_{\ell^{ \pm}}(\rho)$ the function whose graph is the line joining $\left(\ell^{-}, f\left(\ell^{-}\right)\right)$with $\left(\ell^{+}, f\left(\ell^{+}\right)\right)$, see Fig. 1b. 


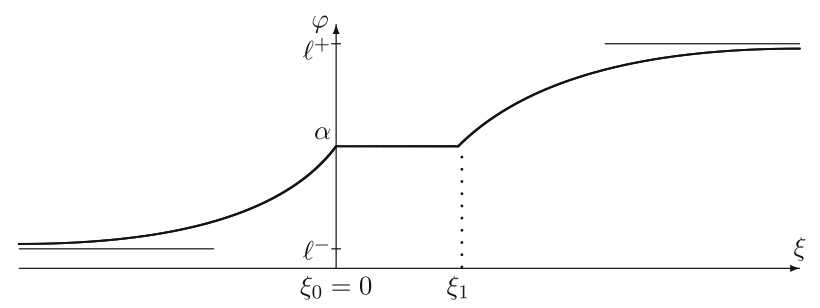

FIG. 2. Under (D1), a profile $\varphi$ in the case $\xi_{1}>0$

Remark 2.1. Assume (f), (D1) and let $\rho$ be a traveling-wave solution to (1.1) with profile $\varphi$ defined in $I$ and speed $c$. We claim that $\varphi$ is classical in every interval $I_{ \pm} \subseteq I$ where $D(\varphi(\xi)) \gtrless 0$ for $\xi \in I_{ \pm}$; indeed, $\varphi \in C^{2}\left(I_{ \pm}\right)$.

As far as the interval $I_{+}$is concerned, this follows by [16, Lemma 2.20 and Theorem. 2.39]. About $I_{-}$, consider the equation

$$
r_{t}+g(r)_{x}=\left(E(r) r_{x}\right)_{x}, \quad r \in[0,1]
$$

with $E(r):=-D(r)$ and $g(r):=-f(r), r \in[0,1]$. Then, $\varphi$ is a traveling-wave solution to (1.1) with speed $c$ iff it is a traveling-wave solution to (2.2) with opposite speed $-c$. Since $E$ and $D$ have opposite signs, we conclude that $\varphi$ is classical also in $I_{-}$and $\varphi \in C^{2}\left(I_{-}\right)$.

For positive diffusivities, profiles are uniquely determined up to a shift [16]. This still holds under (D1), but the loss of uniqueness is more severe as we now show. Assume that (1.1) admits a wavefront solution with profile $\varphi$ and speed $c$, connecting $\ell^{-} \in[0, \alpha)$ with $\ell^{+} \in(\alpha, 1]$. Since $\varphi$ is monotone and continuous by Definition 2.1, then there is a unique $\xi_{0}$, which we can assume to coincide with 0 , such that

$$
\varphi(0)=\alpha \quad \text { and } \quad \varphi(\xi)<\alpha \quad \text { for } \quad \xi<0 .
$$

Similarly, there is a unique $\xi_{1}=\xi_{1} \geq 0$ with

$$
\varphi\left(\xi_{1}\right)=\alpha \quad \text { and } \quad \varphi(\xi)>\alpha \quad \text { for } \quad \xi>\xi_{1} .
$$

If $\xi_{1}>0$, then $\varphi \equiv \alpha$ in $\left[0, \xi_{1}\right]$, see Fig. 2 . The following lemma shows that the case $\xi_{1}>0$ may really occur.

Lemma 2.1. Assume (f) and (D1); suppose that (1.1) admits a wavefront whose profile satisfies (1.5). Then, for every $\xi_{1} \geq 0$ Eq. (1.1) has a wavefront whose profile still satisfies (1.5) and also (2.3), (2.4).

In other words, the loss of uniqueness of the profiles does concern not only shifts but also the "stretching" of the interval $\left[0, \xi_{1}\right], \xi_{1} \geq 0$. The latter loss of uniqueness does not occur if the diffusivity possibly vanishes only at 0 or 1 [16]. Clearly, the profiles mentioned in Lemma 2.1 have the same smoothness. For simplicity, we mainly focus on the case

$$
\xi_{0}=\xi_{1}=0
$$

The case $\xi_{1}>0$ (and related situations) will be discussed at the end of this section.

Now, we state the main results of this paper. 
Theorem 2.1. Assume (f), (D1), $\ell^{-} \in[0, \alpha), \ell^{+} \in(\alpha, 1]$. Equation (1.1) has a wavefront solution whose profile $\varphi$ satisfies (1.5) iff the following three conditions are satisfied:

$$
\begin{aligned}
& \frac{f(\alpha)-f\left(\ell^{-}\right)}{\alpha-\ell^{-}}=\frac{f\left(\ell^{+}\right)-f(\alpha)}{\ell^{+}-\alpha}=: c_{\ell^{ \pm}}, \\
& f(\rho)>s_{\ell^{ \pm}}(\rho) \text { for all } \rho \in\left(\ell^{-}, \alpha\right), \quad f(\rho)<s_{\ell^{ \pm}}(\rho) \text { for all } \rho \in\left(\alpha, \ell^{+}\right), \\
& \frac{D(\rho)}{f(\rho)-s_{\ell^{ \pm}}(\rho)} \in L^{1}\left(I_{\alpha}\right),
\end{aligned}
$$

for some neighborhood $I_{\alpha}$ of $\alpha$, where $s_{\ell^{ \pm}}(\rho)=c_{\ell^{ \pm}}(\rho-\alpha)+f(\alpha)$. The wave speed is $c_{\ell^{ \pm}}$and

$$
f^{\prime}(\alpha) \leq c_{\ell^{ \pm}}
$$

Assume (2.5). Then, $\varphi$ is unique; moreover, $\varphi^{\prime}(\xi)>0$ when $\ell^{-}<\varphi(\xi)<\ell^{+}, \xi \neq 0$, while

$$
\lim _{\xi \rightarrow 0} \varphi^{\prime}(\xi)=\left\{\begin{aligned}
\frac{f^{\prime}(\alpha)-c_{\ell \pm}}{D^{\prime}(\alpha)} & \text { if } D^{\prime}(\alpha)<0, \\
\infty & \text { if } D^{\prime}(\alpha)=0 \text { and } f^{\prime}(\alpha)-c_{\ell^{ \pm}}<0 .
\end{aligned}\right.
$$

This theorem deserves several comments.

(i) We refer to Fig. 1b for the geometric meaning of conditions (2.6), (2.7). If condition (2.7) is satisfied, then $f$ has an inflection point, which, however, does not necessarily coincide with $\alpha$ (see, for instance, the top plots in Fig. 6). Equation (1.1) may have stationary wavefronts; this happens if and only if $f\left(\ell^{-}\right)=f\left(\ell^{+}\right)$and then $f(\alpha)=f\left(\ell^{ \pm}\right)$.

(ii) Condition (2.8) guarantees the existence of profiles from $\ell^{-}$to $\alpha$, and from $\alpha$ to $\ell^{+}$, which reach $\alpha$ for a finite value of $\xi$; they are clearly nonstrictly monotone. We refer to [16, Th. 9.1] for the introduction of such a condition. The fraction in (2.8) is positive in $\left(\ell^{-}, \ell^{+}\right) \backslash\{\alpha\}$ because of (2.7) and (D1), and its denominator vanishes at least at first order at $\alpha$ since $f \in C^{1}$. If $f^{\prime}(\alpha)<c_{\ell^{ \pm}}$, see (2.9), then the assumption $D(\alpha)=0$ in (D1) implies that (2.8) is satisfied because $D \in C^{1}$. As a consequence, condition (2.8) is needed only in case $f^{\prime}(\alpha)=c_{\ell^{ \pm}}$, i.e., when the line $s_{\ell^{ \pm}}$is tangent to the graph of $f$ at $(\alpha, f(\alpha))$.

(iii) Formula (2.10) implies that $\varphi$ is sharp at $\alpha$ if $D^{\prime}(\alpha)=0$ and $f^{\prime}(\alpha)-c_{\ell^{ \pm}}<0$. The case $f^{\prime}(\alpha)-c_{\ell^{ \pm}}=$ $D^{\prime}(\alpha)=0$ is more delicate: in the proof of Theorem 2.1 , we shall show

$$
\lim _{\xi \rightarrow 0^{ \pm}} \varphi^{\prime}(\xi)=\lim _{\sigma \rightarrow \alpha^{ \pm}} \frac{f(\sigma)-\left[f(\alpha)+f^{\prime}(\alpha)(\sigma-\alpha)\right]}{D(\sigma)} .
$$

The existence and the value of that limit depends on the behavior of $f$ and $D$ in a neighborhood of $\alpha$.

(iv) The profile $\varphi$ reaches the value $\ell^{-}$or $\ell^{+}$for a finite $\xi$ if $D /\left(f-s_{\ell^{ \pm}}\right) \in L^{1}\left(I_{\ell^{-}}^{+}\right)$or $D /\left(f-s_{\ell^{ \pm}}\right) \in$ $L^{1}\left(I_{\ell^{+}}^{-}\right)$, where $I_{\ell^{-}}^{+}$and $I_{\ell^{+}}^{-}$are right and left neighborhoods of $\ell^{-}$and $\ell^{+}$, respectively [16, Th. 9.1]. By (D1), this may only happen if either $\ell^{-}=0$ and $D(0)=0$, or $\ell^{+}=1$ and $D(1)=0$, respectively. If $\ell^{-}=0$ and $f^{\prime}(0) \neq c_{\ell^{ \pm}}$, then the solution is sharp in 0 ; the profile is sharp in $\ell^{+}$when $\ell^{+}=1$ and $f^{\prime}(1) \neq c_{\ell^{ \pm}}$. The reasoning is as in [10].

Remark 2.2. Equation (1.1) has wavefront solutions also if we assume $\ell^{+} \in[0, \alpha), \ell^{-} \in(\alpha, 1]$ and replace (D1) in Theorem 2.1 with

( $\widetilde{\mathrm{D} 1}) \quad D \in C^{1}[0,1], D(\rho)<0$ for $\rho \in(0, \alpha)$ and $D(\rho)>0$ for $\rho \in(\alpha, 1)$,

while keeping all the other assumptions. The only difference in this case is that the profile $\varphi$ is decreasing. We omit the proof of such result, which requires a change of variables, and we refer to Sect. 3.1.4 for an application to a model of pedestrian flows. In both cases (D1) and ( $\widetilde{D} 1)$, Eq. (1.1) has wavefront solutions also when the inequalities in (2.7) are reversed. Again, for brevity, we omit this discussion as it is not of interest in models of collective movements.

The results of Theorem 2.1 can be easily extended to the case when $D$ satisfies (D2) $D \in C^{1}[0,1], D(\rho)>0$ for $\rho \in(0, \alpha) \cup(\beta, 1)$ and $D(\rho)<0$ for $\rho \in(\alpha, \beta)$. 


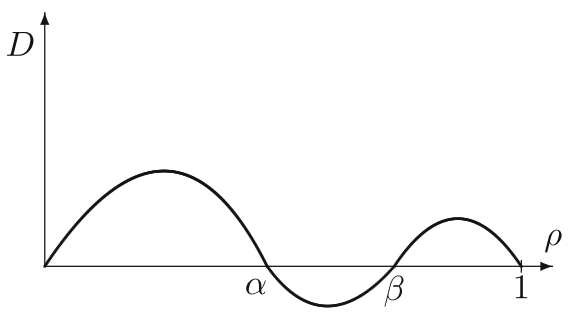

(a)

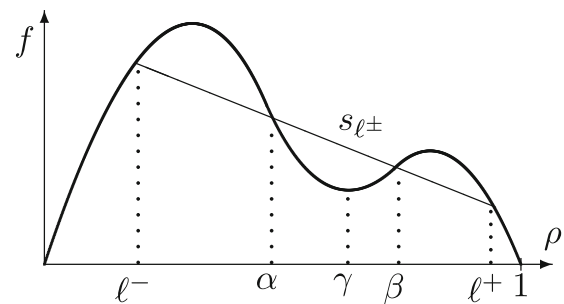

(b)

FIG. 3. a The diffusivity $D$ in case (D2); b The flux function $f$

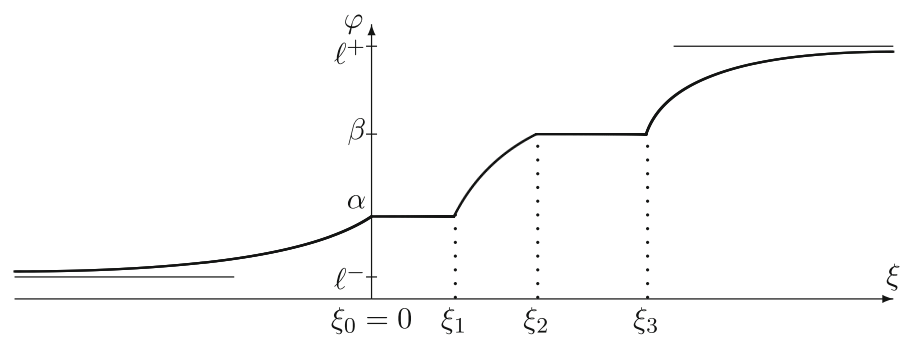

FIG. 4. Under (D2), a profile $\varphi$ in the case $0<\xi_{1}<\xi_{2}<\xi_{3}$

We refer to Fig. 3a for a possible plot of a diffusivity $D$ satisfying (D2).

As in the discussion preceding Lemma 2.1, under (2.3) there are three (unique) values $0 \leq \xi_{1} \leq \xi_{2} \leq \xi_{3}$ such that, see Fig. 4,

$$
\begin{aligned}
& \varphi(\xi)=\alpha \text { for } 0 \leq \xi \leq \xi_{1} \quad \text { and } \quad \alpha<\varphi(\xi)<\beta \quad \text { for } \xi_{1}<\xi<\xi_{2}, \\
& \varphi(\xi)=\beta \text { for } \xi_{2} \leq \xi \leq \xi_{3} \text { and } \quad \varphi(\xi)>\beta \text { for } \xi>\xi_{3} .
\end{aligned}
$$

A result analogous to Lemma 2.1 can be proved; then, for simplicity, we focus on the case

$$
\xi_{0}=\xi_{1}=0, \quad \xi_{2}=\xi_{3} .
$$

For brevity, in the next statement we collect only the most important facts about case (D2); other results as in Theorem 2.1 follow in a direct way. The proof is omitted.

Theorem 2.2. Assume (f) and (D2); let $\ell^{-} \in[0, \alpha)$ and $\ell^{+} \in(\beta, 1]$. Equation (1.1) admits a wavefront solution whose profile $\varphi$ satisfies (1.5) if and only if the following three conditions are satisfied for some neighborhoods $I_{\alpha}$ of $\alpha$ and $I_{\beta}$ of $\beta$, respectively:

$$
\begin{aligned}
& \frac{f(\alpha)-f\left(\ell^{-}\right)}{\alpha-\ell^{-}}=\frac{f(\beta)-f(\alpha)}{\beta-\alpha}=\frac{f\left(\ell^{+}\right)-f(\beta)}{\ell^{+}-\beta}=: c_{\ell^{ \pm}}, \\
& f(\rho)>s_{\ell^{ \pm}}(\rho) \text { if } \rho \in\left(\ell^{-}, \alpha\right) \cup\left(\beta, \ell^{+}\right), \quad f(\rho)<s_{\ell^{ \pm}}(\rho) \text { if } \rho \in(\alpha, \beta), \\
& \frac{D(\rho)}{f(\rho)-s_{\ell^{ \pm}}(\rho)} \in L^{1}\left(I_{\alpha}\right) \cap L^{1}\left(I_{\beta}\right) .
\end{aligned}
$$

Under (2.13) the profile is unique; moreover, $\varphi^{\prime}(\xi)>0$ when $\ell^{-}<\varphi(\xi)<\ell^{+}, \xi \neq 0, \xi \neq \xi_{2}$.

We refer to Fig. 3b for a geometrical interpretation of conditions (2.14) and (2.15). At last, we consider the family of equations

$$
\rho_{t}+f(\rho)_{x}=\left(\varepsilon D(\rho) \rho_{x}\right)_{x}, \quad t \geq 0, x \in \mathbb{R},
$$


depending on $\varepsilon \in(0,1]$; we are interested in the limit as $\varepsilon \rightarrow 0^{+}$of the wavefronts profiles $\varphi_{\varepsilon}$. This subject falls in the much more general issue of the convergence of solutions $\rho_{\varepsilon}$ of (2.17) to a solution $\rho$ of (1.6). If $D>0$, a positive answer is provided in [25]; see $[11, \S 6]$ for more information. The case $D \geq 0$ was first considered in [43]; see [8] for a short proof in the presence of source terms and updated references. To the best of our knowledge, no analogous information is known when $D$ changes sign. We provide now a convergence result concerning wavefronts.

About (2.17), we assume (f), (D1), (2.6), (2.7); for sake of simplicity, we also suppose

$$
D^{\prime}(\alpha)<0 \text {. }
$$

By Theorem 2.1, for every $\ell^{-} \in[0, \alpha), \ell^{+} \in(\alpha, 1]$ and $\varepsilon \in(0,1]$, there exist unique profiles $\varphi_{\varepsilon}(\xi)$ of wavefronts of (2.17) satisfying (2.5). All of them have the same speed $c_{\ell^{ \pm}}$.

Theorem 2.3. We assume (f), (D1) and consider $\ell^{-} \in[0, \alpha), \ell^{+} \in(\alpha, 1]$; we also assume (2.6)-(2.8) as well as (2.18). Let $\varphi_{\varepsilon}$ be the unique profiles of wavefront solutions to (2.17) satisfying (1.5) and (2.5). Then

$$
\lim _{\varepsilon \rightarrow 0+} \varphi_{\varepsilon}(\xi)=: \varphi_{0}(\xi)= \begin{cases}\ell^{-} & \text {if } \xi<0, \\ \ell^{+} & \text {if } \xi>0 .\end{cases}
$$

The convergence is uniform in every interval $(-\infty,-\delta)$ and $(\delta,+\infty)$ with $\delta>0$.

The results of Theorem 2.1 can be easily reformulated to cover the case $\xi_{1}>0$; in particular, formula (2.10) corresponds to the limit for $\xi \rightarrow 0^{-}$, while a completely analogous result holds for the limit $\xi \rightarrow \xi_{1}^{+}$. Assume $D^{\prime}(\alpha)<0$; if $\xi_{1}=0$, then $\varphi$ is of class $C^{1}$ at $\xi=0$, by $(2.10)$, while if $\xi_{1}>0$, this does not hold unless $f^{\prime}(\alpha)=c_{\ell^{ \pm}}$. Analogous remarks apply to Theorem 2.2 in the case $\xi_{1}>0$ and $\xi_{2}<\xi_{3}$. About Theorem 2.3, consider again a family $\varphi_{\varepsilon}$ of profiles for $(2.17)$ but suppose that there exists $\xi_{1}>0$ that does not depend on $\varepsilon$ such that $\alpha=\varphi_{\varepsilon}\left(\xi_{1}\right)<\varphi_{\varepsilon}(\xi)$ for $\xi>\xi_{1}$. Arguing as in the proof of Theorem 2.3, we deduce that

$$
\lim _{\varepsilon \rightarrow 0+} \varphi_{\varepsilon}(\xi)=: \varphi_{0, \xi_{1}}(\xi)= \begin{cases}\ell^{-} & \text {if } \xi \in(-\infty, 0), \\ \alpha & \text { if } \xi \in\left(0, \xi_{1}\right), \\ \ell^{+} & \text {if } \xi \in\left(\xi_{1}, \infty\right) .\end{cases}
$$

Theorem 2.3 allows us to comment on the above results from the hyperbolic point of view. The function $\rho_{0}(x, t)=\varphi_{0}\left(x-c_{\ell^{ \pm}} t\right)$ is a weak (distributional) solution to Eq. (1.1) because the Rankine-Hugoniot conditions are satisfied.

Conditions analogous to (2.7) are well known in the hyperbolic setting [6, Theorem 4.4]. In particular, the discontinuous solution $\rho_{0}$ is not entropic; it is so the analogous solution joining $\ell^{-}$with $\alpha$. Referring to the case depicted in Fig. 1b, the Lax inequality $f^{\prime}\left(\ell^{-}\right)>c_{\ell^{ \pm}}$is satisfied while $c_{\ell^{ \pm}}>f^{\prime}\left(\ell^{+}\right)$fails: the shock is compressive on the left and undercompressive on the right. However, even if $\rho_{0}$ is not entropic, Theorem 2.1 shows that it has a viscous profile, where "viscous" refers to a negative diffusivity in the nonentropic part of the solution; such a wave is unstable in the sense of [6, Rem. 4.7]. Different scenarios are also possible: for instance, the one-sided sonic case $c_{\ell^{ \pm}}=f^{\prime}\left(\ell^{+}\right) \neq f^{\prime}\left(\ell^{-}\right)\left(\right.$or $\left.c_{\ell^{ \pm}}=f^{\prime}\left(\ell^{-}\right) \neq f^{\prime}\left(\ell^{+}\right)\right)$ or even the doubly sonic case $c_{\ell^{ \pm}}=f^{\prime}\left(\ell^{ \pm}\right)$, see Fig. 5. The former case has been considered in [9] (see cases (R1) and (R3)(a) there) in the framework of nonclassical shocks [27]. Generically, there is a sheaf of lines through $(\alpha, f(\alpha))$ that intersect the graph of $f$ in two further points and then a one-parameter family of end states $\left(\ell^{-}, \ell^{+}\right)$for which $(2.7)$ is satisfied.

In the case $\xi_{1}>0$, the function $\rho_{0, \xi_{1}}(x, t)=\varphi_{0, \xi_{1}}\left(x-c_{\ell^{ \pm}} t\right)$ is a weak solution to (1.6) because its jumps satisfy the Rankine-Hugoniot conditions. However, the entropy condition fails, as discussed above.

About Theorem 2.2, consider, for instance, the case depicted in Fig. 3b. The discontinuous solution $\rho$ whose profile joins $\ell^{-}$on the left to $\ell^{+}$on the right with a jump propagating with velocity $c_{\ell^{ \pm}}$is still nonentropic. Both Lax inequalities $f^{\prime}\left(\ell^{-}\right)>c_{\ell^{ \pm}}>f^{\prime}\left(\ell^{+}\right)$are satisfied, but this does not imply that the solution is entropic because the flux is not convex [6, Remark 4.7]. Also shock waves connecting the states 


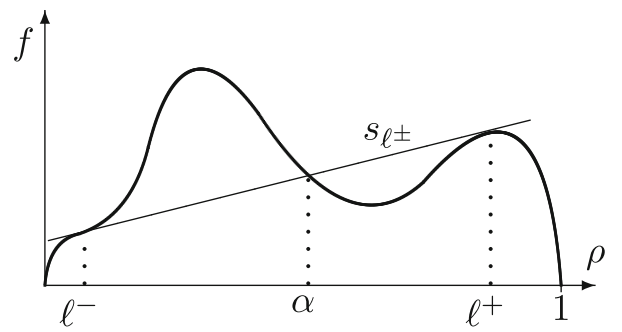

Fig. 5. A flux function $f$ in the doubly sonic case

$\ell^{-}$and $\ell^{+}$as in Fig. 3b have been considered in [9] (see case (R3)(b) there). Notice that in the framework of Theorem 2.2 we generically have a unique pair of end states $\ell^{ \pm}$.

In [9] the (hyperbolic) zone of "panic" is modeled by the interval $(\gamma, 1]$, where $\gamma$ is a local minimum of $f$, see Fig. $3 \mathrm{~b}$; the zone of aggregation (where $D<0$ ) is $(\alpha, \beta)$. The balance between panic and aggregation can be explained by our diffusive model as follows. When the density is in the interval $(\alpha, \gamma)$, panic has not yet emerged, but the crowd shows an aggregative behavior to face the perceived danger. This behavior persists even after the threshold $\gamma$ is trespassed if the density is not exceeding, namely in the interval $(\gamma, \beta)$. Values of $\rho \in(\beta, 1)$ are unbearable and push the crowd to (slightly) diffuse again.

\section{Applications to collective movements}

In this section, we provide some examples concerning the modeling of vehicular traffic flows or crowds dynamics. In these cases, assumption (f) specializes to $[28,36]$

(fcm) $f(\rho)=\rho v(\rho)$, with $v \in C^{1}[0,1], v(\rho) \geq 0$ for $\rho \in[0,1)$ and $v(1)=0$.

Here, $v$ is the velocity; from a modeling point of view, $v$ may vanish once [9] in $[0,1)$ and is decreasing at least in a right neighborhood of 0 . About $D$, the properties $D(0)=D(1)=0$ would be desirable $[4,7]$. We now list the main models of $D$ occurring in the literature.

In [32], the author proposed the expression

$$
D(\rho)=-\delta \rho v^{\prime}(\rho)-\tau \rho^{2} v^{\prime}(\rho)^{2}=-\rho v^{\prime}(\rho)\left(\delta+\tau \rho v^{\prime}(\rho)\right),
$$

where $\delta>0$ is an anticipation distance and $\tau>0$ a reaction time; see also [19] for these parameters in a kinetic framework. The safety velocity $\delta / \tau=: v^{s}$ is the velocity needed to cover the distance $\delta$ in the time $\tau$. Under this notation, (3.1) can be written as

$$
D(\rho)=-\tau \rho v^{\prime}(\rho)\left(v^{s}+\rho v^{\prime}(\rho)\right) .
$$

If $\bar{v}=\max _{\rho \in[0,1]} v(\rho)$, then a natural requirement is

$$
\bar{v} \leq v^{s}
$$

In crowds dynamics, the parameter $\tau$ is very small and may be dropped [7]; this leads to

$$
D(\rho)=-\delta \rho v^{\prime}(\rho) .
$$

The case $\delta=\delta(\rho)=h v^{2}(\rho), h>0$, in (3.1) is also proposed in [32]. If it is so, (3.1) becomes

$$
D(\rho)=-\rho v^{\prime}(\rho)\left(h v^{2}(\rho)+\tau \rho v^{\prime}(\rho)\right) .
$$

In the case of pedestrian flows, instead of (3.5) one may consider [7, Figure 4]

$$
D(\rho)=-\rho v^{\prime}(\rho)\left(h v(\rho)+\tau \rho v^{\prime}(\rho)\right) .
$$

For simplicity, in (3.5) and (3.6) we have taken $\tau \geq 0$ to be independent of $\rho$. 
Several different models for $D$ follow by a kinetic approximation. In [20], the authors motivate the occurrence of stop and go waves precisely by the presence of zones with negative diffusivities. For instance, in the case of a kinetic model with two microscopic velocities $0 \leq \xi_{1}<\xi_{2}$, one deduces from [20] that $D(\rho)=\tau\left(\left(\xi_{1}+\xi_{2}\right) q^{\prime}(\rho)+\xi_{1} \xi_{2}-\left[q^{\prime}(\rho)\right]^{2}\right)$, where $q(\rho)=\rho v(\rho)$ is the hyperbolic flow and $\tau>0$ a reaction time. In our model, $v$ ranges from 0 to $\bar{v}$ and then we take $\xi_{1}=0, \xi_{2}=\bar{v}$; we deduce

$$
D(\rho)=\tau\left(\rho v^{\prime}(\rho)+v(\rho)\right)\left(\bar{v}-\rho v^{\prime}(\rho)-v(\rho)\right) .
$$

We limit the examples below to the simpler diffusivities (3.1), (3.4)-(3.6).

\subsection{The case (D1)}

We now investigate when assumptions (fcm), (D1), (2.7), (2.8) are satisfied according to the choice of $v$, when $D$ is chosen as in (3.1), (3.4)-(3.6). Proofs are deferred to Sect. 5.

\subsubsection{Two negative results.}

Lemma 3.1. If $D(\rho)=-a(\rho) v^{\prime}(\rho)$ with $a>0$ in $(0,1) \backslash\{\alpha\}$, then assumptions (fcm) and (D1) cannot hold together.

Proof. By (D1) we have $v^{\prime}(\rho)<0$ if $\rho \in(0, \alpha)$ and $v^{\prime}(\rho)>0$ if $\rho \in(\alpha, 1)$. Since $v$ increases in $(\alpha, 1)$, by $v(1)=0$ we deduce $v(\alpha)<0$, which contradicts the positivity of $v$.

Then neither (3.4) nor (3.5) with $\tau=0$ satisfy both (fcm) and (D1). We consider (3.1).

Lemma 3.2. Assume $D$ is given by (3.1). If $f$ and $D$ satisfy (fcm) and (D1), respectively, then $v$ must be decreasing. The simple expressions

$$
v(\rho)=\frac{\delta}{\alpha \tau}(1-\rho), \quad v(\rho)=\frac{\delta}{2 \alpha \tau}(1-\rho)(1+2 \alpha-\rho) .
$$

imply both (fcm) and (D1). Conditions (2.7) and (3.3) fail in both cases.

3.1.2. Vehicular flows: the case of quadratic speed. The two previous lemmas show that the expressions (3.1), (3.4) and (3.5) with $\tau=0$ never or difficultly satisfy conditions (fcm), (D1) and (2.7) for some simple velocity $v$. Then, we focus on the case (3.5) with $\tau>0$, where we provide positive results. In order that $D(1)=0$ holds, we need $v$ vanishes at second order at $\rho=1$; then, we consider

$$
v(\rho)=\bar{v}(1-\rho)^{2},
$$

for $\bar{v}>0$. The function $f$ has an inflection point at $\tilde{\rho}=2 / 3$; it is strictly concave in $[0, \tilde{\rho})$ and strictly convex in $(\tilde{\rho}, 1]$. We have

$$
D(\rho)=2 h \bar{v}^{3} \rho(1-\rho)^{2}\left[(1-\rho)^{3}-\sigma \rho\right], \quad \sigma:=\frac{2 \tau}{h \bar{v}}>0 .
$$

Lemma 3.3. Let $v$ be given by (3.9) and $D$ by (3.5) with $\tau \neq 0$, see (3.10). Then, $D$ satisfies (D1) for any positive $\bar{v}, h, \tau$ such that $\alpha=\alpha(\bar{v}, h, \tau)$ is the unique root in $(0,1)$ of

$$
(1-\alpha)^{3}=\sigma \alpha \text {. }
$$

The function $\alpha(\bar{v}, h, \tau)$ covers the interval $(0,1)$ for $\tau, h, \bar{v} \in(0, \infty)$. If $\tau, h, \bar{v}$ are such that $\alpha(\bar{v}, h, \tau) \in$ $(1 / 2,1)$, then there are infinitely many pairs $\left(\ell^{-}, \ell^{+}\right)$such that $(2.6)-(2.8)$ hold.

The point $\alpha$ does not need to be an inflection point of $f$; this happens, in the example above, if $\alpha=2 / 3$. Top plots in Fig. 6 give an illustration of the example considered in Lemma 3.3 for real-world data [32]. There, we use dimensional variables.

The previous example can be generalized to $v(\rho)=\bar{v}\left(1-\rho^{p}\right)^{q}$, for $p>0, q>1$. The condition $q>1$ is needed in order that $f$ has an inflection point $\left(\right.$ at $\left.\rho=\left(\frac{1+p}{1+p q}\right)^{1 / p}\right)$ in $(0,1)$. 

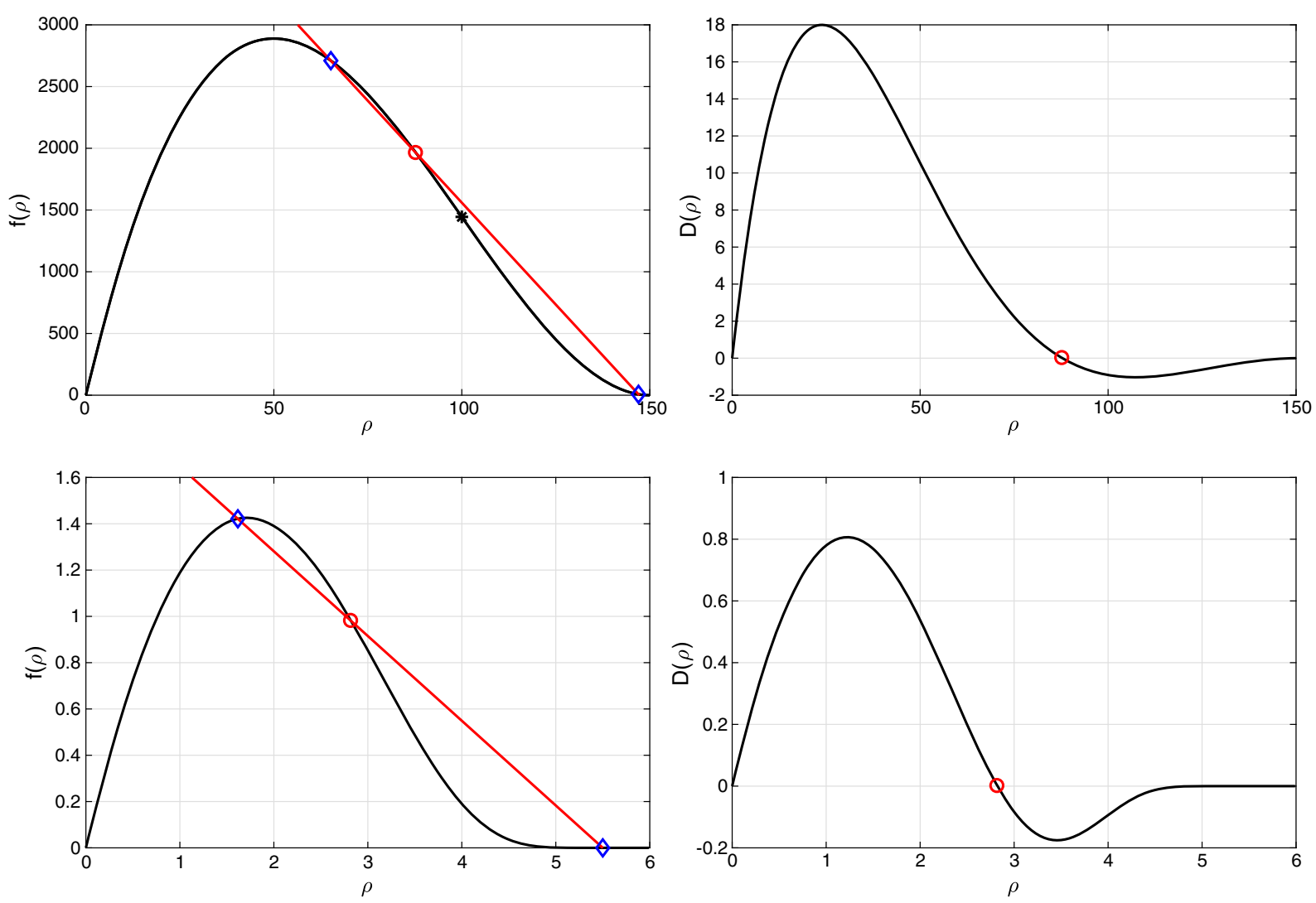

FIG. 6. Plots of flows (left column) and diffusivities (right column); the end states are depicted with diamonds. Top: Here, $v(\rho)=\bar{v}\left(1-\frac{\rho}{\rho}\right)^{2}, D$ as in $(3.5), \bar{\rho}=150 \mathrm{cars} / \mathrm{km}, \bar{v}=130 \mathrm{~km} / \mathrm{h}, \tau=2 \mathrm{~s}, h=1 / 15,800 \mathrm{~h}^{2} / \mathrm{km}$, see [32]. An empty circle localizes $\alpha \sim 88$, an asterisk the inflection point of $f$, which is 100 . For $\ell^{+}=147$, we find $\ell^{-} \sim 65$. Bottom: Here, $v$ is as in $(3.12)_{2}, a=0, D$ as in (3.6), $\bar{\rho}=6$ pedestrian $/ \mathrm{m}, \bar{v}=1.7 \mathrm{~m} / \mathrm{s}, \tau=0.5 \mathrm{~s}, h=1.5, \gamma=1.788$. Data are taken from [42], in the case of rush-hours pedestrian flows

3.1.3. Pedestrian flows: the case of exponential speed. We now consider the two following laws for pedestrian flows in normalized variables [42]:

$$
v(\rho)=\bar{v}\left(1-e^{\gamma\left(1-\frac{1}{\rho}\right)}\right) \quad \text { and } \quad v(\rho)= \begin{cases}\bar{v} & \text { if } \rho \leq a, \\ \bar{v} e^{\gamma \frac{a-\rho}{1-\rho}} & \text { if } \rho>a,\end{cases}
$$

where $\gamma>0, \bar{v}>0$ and $0 \leq a<1$ is a critical density that separates free from congested flow. Both functions are extended by continuity at 0 and 1 , respectively. Law $(3.12)_{1}$ is called Kladek formula. The law $(3.12)_{2}$ with $a>0$ does not satisfies (fcm) because $v \notin C^{1}$, unless we consider it only in $(a, 1)$ or we set $a=0$.

In case $(3.12)_{1}$, the function $f$ is strictly concave; then, (2.7) cannot hold and so we focus on case $(3.12)_{2}$. Assume $a \gamma<2$; then $f$ is strictly concave (convex) in $[a, \tilde{\rho})$ (in $\left.(\tilde{\rho}, 1]\right)$ for

$$
\tilde{\rho}=\frac{1}{1+\frac{\gamma}{2}(1-a)} \in(a, 1) .
$$

If $D$ is as in (3.1), then $D(1)=0$ but $D$ is strictly positive in a left neighborhood of 1 ; hence, condition (D1) fails. If $D$ is as in (3.4), then $D(0)=D(1)=0$ and $D(\rho)>0$ if $\rho \in(a, 1)$. 
Assume $D$ is as in (3.5). Then, $D(1)=0$ and $D$ vanishes at the point $\alpha$ defined by

$$
\frac{\tau \gamma(1-a)}{h \bar{v}} \frac{\alpha}{(1-\alpha)^{2}}=e^{\gamma \frac{a-\alpha}{1-\alpha}}
$$

provided that $a<\frac{1}{1+\bar{\sigma}}$, for $\bar{\sigma}=\tau \gamma /(h \bar{v})$. In this case, assumptions (f) and (D1) are satisfied and conditions (2.6), (2.7) can be numerically checked for fixed end states $\ell^{ \pm}$. Plots are similar to those in the top row of Fig. 6 and are omitted.

Now, we discuss (2.8). If $\tilde{\rho} \neq \alpha$ then, by arguing as in the last part of the proof of Lemma 3.3, we deduce $f^{\prime}(\tilde{\rho})<c_{\ell^{ \pm}}$and $(2.8)$ is satisfied by comment (ii) to Theorem 2.1. The case $\tilde{\rho}=\alpha$ cannot be treated so easily as the analogous case in Lemma 3.3, because the analytic expression of the velocity is more complicated. However, we claim that, as in Lemma 3.3, there are infinitely many end states $\ell^{ \pm}$such that (2.6), (2.7) and (2.8) are satisfied.

To prove the claim, assume $\ell^{ \pm}$satisfies (2.6) and (2.7). For $\mu$ close to 0 consider the line

$$
s_{\mu}(\rho)=\frac{f(\alpha)-f\left(\ell^{-}+\mu\right)}{\alpha-\left(\ell^{-}+\mu\right)}(\rho-\alpha)+f(\alpha)
$$

passing through $\left(\ell^{-}+\mu, f\left(\ell^{-}+\mu\right)\right)$ and $(\alpha, f(\alpha))$ and define $F(\rho, \mu):=f(\rho)-s_{\mu}(\rho)$. Then, $F\left(\ell^{+}, 0\right)=0$ and $F_{\mu}\left(\ell^{+}, 0\right)>0$ because $f$ is strictly concave in $[a, \tilde{\rho}]$. By the implicit function theorem, there exists a function $y=y(\mu)$, which is defined in a neighborhood of 0 , such that the line $s_{\mu}$ intersects the graph of $f$ in the further point $\left(\ell^{+}+y(\mu), f\left(\ell^{+}+y(\mu)\right)\right.$. The pairs of end states $\ell^{-}+\mu$ and $\ell^{+}+y(\mu)$, one pair at most excepted, satisfy $(2.6)-(2.8)$.

At last, consider again $v$ given by $(3.12)_{2}$ with $a=0$ but $D$ as in (3.6). In this case, for real-world data, conditions (2.6), (2.7) are satisfied if $\tau$ is sufficiently small, see the bottom plots in Fig. 6 . For higher values of $\tau$, the point $\alpha$ falls on the left of the maximum point of $f$. About (2.8), we can argue as in the previous example.

3.1.4. The case of negative-to-positive diffusivity. Consider the velocity in $(3.12)_{2}$ with $0<a<1, f$ as in (fcm), $D$ given by (3.1), and assume (3.3). Recall the notation $v^{s}=\delta / \tau$ and $w:=\bar{v} / v^{s} \in(0,1]$. First, we claim that if

$$
\gamma>\frac{1-a}{a w} \quad \text { and } \quad \gamma \geq \frac{1+a}{a}
$$

then there is $\alpha \in(a, 1)$ such that $D<0$ in $(a, \alpha)$ and $D>0$ in $(\alpha, 1)$. To prove the claim, first notice that $(3.14)_{2}$ implies $\gamma>2$ because $0<a<1$. Moreover, for $\rho \in(a, 1)$ we have

$$
D(\rho)=\frac{\tau \bar{v}^{2} \gamma^{2}(1-a)^{2}}{(1-\rho)^{4}} \rho^{2} e^{\gamma \frac{a-\rho}{1-\rho}} \quad \text { for } \quad h(\rho):=\frac{1}{\gamma w(1-a)} \frac{(1-\rho)^{2}}{\rho}-e^{\gamma \frac{a-\rho}{1-\rho}} .
$$

We pointed out below (3.13) that $h(\rho)>0$ in a left neighborhood of 1 ; moreover $(3.14)_{1}$ is equivalent to $h(a)<0$. Then, there is $\alpha \in(a, 1)$ such that $h(\alpha)=0$, implying

$$
\frac{1}{\gamma w(1-a)} \frac{(1-\alpha)^{2}}{\alpha}=e^{\gamma \frac{a-\alpha}{1-\alpha}} .
$$

We compute $h^{\prime}(\alpha)=\frac{1}{\gamma w(1-a) \alpha^{2}} \psi(\alpha)$, for $\psi(\rho):=\rho^{2}+\gamma(1-a) \rho-1$ and $\rho \in(a, 1)$. By $(3.14)_{2}$ we deduce $\psi(a)=(1-\gamma) a^{2}+\gamma a-1 \geq 0$. Moreover, $\psi^{\prime}(\rho)>0$ for $\rho>0$, and so $\psi(\alpha)>0$ whatever $\alpha \in(a, 1)$ is. Then, $h^{\prime}(\alpha)>0$ and so $\alpha$ is unique. This proves our claim.

Hence, $D$ satisfies assumption $(\widetilde{\mathrm{D} 1})$ in the interval $[a, 1]$. Assume $a \gamma<2$; it is always possible to choose $a$ satisfying this condition and $(3.14)_{2}$ because $a<1$. Then, $f(\rho)$ is concave in $(a, \tilde{\rho})$ and convex in $(\tilde{\rho}, 1)$, see the discussion preceding (3.13). Assume now that conditions (2.6) and (2.7) are satisfied and $a \leq \ell^{+}<\alpha<\ell^{-} \leq 1$. If $\alpha \neq \tilde{\rho}$, then condition (2.8) follows by comment (ii) to Theorem 2.1 while the case $\alpha=\tilde{\rho}$ can be discussed as in the examples above. Then, Remark 2.2 applies: Eq. (1.1) admits a wavefront solution with profile $\varphi$ satisfying $\varphi( \pm \infty)=\ell^{ \pm}$ 


\subsection{The case (D2)}

As one may guess from the examples provided in case (D1), in case (D2) it is hard to provide explicit and realistic expressions of velocity laws $v(\rho)$ such that (fcm), (D2) and (2.14), (2.15) are satisfied when $D$ is chosen as in (3.1)-(3.6). We point out this difficulty by considering just one case, analogous to the one in Lemma 3.1. Assume

$$
D(\rho)=-a(\rho) v^{\prime}(\rho), \quad a>0 \text { in }(0,1) \backslash\{\alpha, \beta\} .
$$

This covers both (3.4) and (3.5) with $\tau=0$. The simplest prototype for $v$ in order that (D2) holds and $v(1)=0$ is that $v$ is a third-order polynomial vanishing at 1 , namely

$$
v^{\prime}(\rho)=-(\rho-\alpha)(\rho-\beta), \quad v(1)=0 .
$$

We dropped any positive multiplicative constant in the right-hand side of (3.17).

Lemma 3.4. Let $D$ be as in (3.16) with $v$ as in (3.17). Assumptions (fcm), (D2) hold iff $(\alpha, \beta) \in(0,1)^{2}$ satisfy $3 \beta-2<\alpha<\beta$. In this case, the line $s_{\ell^{ \pm}}$has positive slope.

Lemma 3.4 shows that the case depicted in Fig. 3b (with $s_{\ell^{ \pm}}$having negative slope) never takes place if $D, v$ are as in (3.16), (3.17) and (D2) is satisfied. This diffusivity cannot provide viscous profiles to the shock waves introduced in [9], case $(\mathrm{R} 3)(b)$.

\section{A preliminary result}

In this section, we state a result about the existence of wavefront solutions for (1.1) in intervals $(a, b)$ where $D$ has constant sign. This is well known [16] if $D>0$ while the case $D<0$ is deduced from the previous one. A short proof is given for completeness. The line

$$
s(\rho):=\frac{f(b)-f(a)}{b-a}(\rho-b)+f(b), \quad \rho \in \mathbb{R}
$$

plays a fundamental role in this discussion. Indeed, the existence of wavefront solutions with profile $\varphi$ from $a$ to $b$ depends both on the sign of $D$ and on the behavior of the graph of $f(\rho)$ with respect to the line $s(\rho)$. Moreover, $\varphi$ satisfies the boundary conditions

$$
\varphi(-\infty)=a, \varphi(+\infty)=b \quad \text { or } \quad \varphi(-\infty)=b, \varphi(+\infty)=a .
$$

Theorem 4.1. Let $f, D \in C^{1}[a, b]$. Assume

(a) $D(\rho)>0$ in $(a, b)$; then

(a1) Equation (1.1) has a wavefront of profile $\varphi$ with (4.1) 1 iff $f(\rho)>s(\rho)$ for $\rho \in(a, b)$;

(a2) Equation (1.1) has a wavefront of profile $\varphi$ with $(4.1)_{2}$ iff $f(\rho)<s(\rho)$ for $\rho \in(a, b)$;

(b) $D(\rho)<0$ in $(a, b)$; then

(b1) Equation (1.1) has a wavefront of profile $\varphi$ with $(4.1)_{2}$ iff $f(\rho)>s(\rho)$ for $\rho \in(a, b)$;

(b2) Equation (1.1) has a wavefront of profile $\varphi$ with $(4.1)_{1}$ iff $f(\rho)<s(\rho)$ for $\rho \in(a, b)$.

The profile $\varphi$ is unique (up to shifts), $\varphi \in C^{2}(J), J:=\{\xi \in \mathbb{R}: a<\varphi(\xi)<b\}$, and $\varphi^{\prime}(\xi)>0\left(\varphi^{\prime}(\xi)<0\right)$ for $\xi \in J$ in cases (a1) and (b2) (resp., in cases (a2) and (b1)); the wave speed is $c=(f(b)-f(a)) /(b-a)$. Moreover, the following holds true:

(i) $\varphi$ reaches a, for some $\xi_{a} \in \mathbb{R}$, if and only if $D /(f-s) \in L^{1}\left(I_{a}^{+}\right)$for some right neighborhood $I_{a}^{+}$ of a. Then, $\varphi(\xi)=a$ for $\xi \leq \xi_{a}$ (for $\xi \geq \xi_{a}$ ) in cases (a1), (b2) (resp., in cases (a2), (b1)) and $\lim _{\xi \rightarrow \xi_{a}} D(\varphi(\xi)) \varphi^{\prime}(\xi)=0$.

(ii) $\varphi$ reaches $b$, for some $\xi_{b} \in \mathbb{R}$, if and only if $D /(f-s) \in L^{1}\left(I_{b}^{-}\right)$for some left neighborhood $I_{b}^{-}$ of b. Then, $\varphi(\xi)=b$ for $\xi \geq \xi_{b}$ (for $\xi \leq \xi_{b}$ ) in cases (a1), (b2) (resp., in cases (a2), (b1)) and $\lim _{\xi \rightarrow \xi_{b}} D(\varphi(\xi)) \varphi^{\prime}(\xi)=0$. 
(iii) In the other cases $J=\mathbb{R}$ and $\lim _{\xi \rightarrow \pm \infty} \varphi^{\prime}(\xi)=0$.

Proof. We first prove cases (a1), (a2) by means of [16, Theorem 9.1]; then, we reduce items (b1), (b2) to (a1), (a2), respectively, by a suitable change of variables. We provide full details for case (a1) while we sketch the proofs in the other cases.

Preliminarly, consider Eq. (2.2) for $g, E \in C^{1}[0,1]$. If $E>0$ in $(0,1)$ and $g(0)=0$, then by [16, Theorem 9.1] there exists a wavefront from 1 to 0 of Eq. (2.2) if and only if $g(r) / r<g(1)$ for $r \in(0,1)$; the speed of the wavefront is $g(1)$.

(a1) We denote

$$
E(r):=D(b-(b-a) r), \quad g(r):=-\frac{f(b-(b-a) r)-f(b)}{b-a}, \quad r \in[0,1] .
$$

Notice that $E>0$ in $(0,1)$ and $g(0)=0$. The condition $f(\rho)>s(\rho), \rho \in(a, b)$, is equivalent to $g(r) / r<g(1), r \in(0,1)$; if it holds, by [16, Theorem 9.1] Eq. (2.2) with $g$ and $E$ as in (4.2) has a wavefront with profile $\psi \in C^{2}(I)$, where $I:=\{\xi \in \mathbb{R}: 0<\psi(\xi)<1\}$. Moreover, we have $\psi(-\infty)=1, \psi(+\infty)=0$, $\psi^{\prime}(\xi)<0$ for $\xi \in I$ and $c$ is as above. At last, by Remark 2.1, $\psi$ satisfies $\left(E(\psi) \psi^{\prime}\right)^{\prime}+(c \psi-g(\psi))^{\prime}=0$ in $I$. Then, we define $\varphi(\xi):=b-(b-a) \psi(\xi), \xi \in \mathbb{R}$. We have $\varphi \in C^{2}(J)$, it satisfies (1.4) in $J,(4.1)_{1}$ and $\varphi^{\prime}(\xi)>0$ for $\xi \in J$. Moreover, since $\psi$ satisfies properties analogous to (i)-(iii) by [16, Theorem 9.1], properties (i)-(iii) for $\varphi$ easily follow (see also [10, Theorem 3.2]). Then, Eq. (1.1) has a wavefront with profile $\varphi$ and speed $c$. The converse implication follows directly.

(a2) We denote $E(r):=D((b-a) r+a), g(r):=[f((b-a) r+a)-f(a)] /(b-a)$ for $r \in[0,1]$. Then $E>0$; moreover, $f(\rho)<s(\rho)$ for $\rho \in(a, b)$ iff $g(r) / r<g(1)$ for $r \in(0,1)$. Equation (2.2) with $E$ and $g$ as above has a wavefront with profile $\psi \in C^{2}(I)$ as in case (a1) iff $f(\rho)<s(\rho)$ for $\rho \in(a, b)$. Then $\varphi(\xi):=(b-a) \psi(\xi)+a$ is the profile of a wavefront solution to Eq. (1.1) with speed $c$ and $\varphi^{\prime}(\xi)<0$ for $\xi \in J$.

(b1) We denote $E(r):=-D(b-(b-a) r), g(r):=[f(b-(b-a) r)-f(b)] /(b-a)$ for $r \in[0,1]$. Then $E>0$ in $(0,1)$ and $g(r)>g(1) r, r \in(0,1)$, is equivalent to $f(\rho)>s(\rho), \rho \in(a, b)$. By (a1), Eq. (2.2) has a wavefront with profile $\psi$ satisfying $\psi(-\infty)=0, \psi(+\infty)=1$ iff $f(\rho)>s(\rho)$ for $\rho \in(a, b)$. The speed is given by $-c, \psi \in C^{1}(I), \psi^{\prime}(\xi)>0$ for $\xi \in I$ and $\psi$ satisfies (i)-(iii). Then $\varphi(\xi):=b-(b-a) \psi(\xi)$ satisfies $(4.1)_{2}$ and it is the profile of a wavefront solution to (1.1) with speed $c$ and $\varphi^{\prime}(\xi)<0$ for $\xi \in J$.

(b2) We denote $E$ and $g$ as in case (b1). Then $E>0$ in $(0,1)$ and $g(r)<g(1) r, r \in(0,1)$, is equivalent to $f(\rho)<s(\rho), \rho \in(a, b)$. By (a2), Eq. (2.2) has a wavefront with profile $\psi$ and speed $-c$ iff $f(\rho)<s(\rho)$, $\rho \in(a, b)$; moreover, $\psi \in C^{2}(I)$ as in case (a1) and $\psi$ satisfies (i)-(iii). Then $\varphi(\xi):=b-(b-a) \psi(\xi)$, $\xi \in \mathbb{R}$, satisfies $(4.1)_{1}, \varphi^{\prime}(\xi)>0$ for $\xi \in J$, it is the profile of a wavefront solution to (1.1) with speed $c$.

Example 4.1. We refer in the following to the notation in $(\mathrm{fcm})$ introduced in Sect. 3.

Consider $v(\rho)=1-\rho$; then $f$ is strictly concave. If $D$ is given by $(3.1)$, then $D(\rho)=\rho(\delta-\tau \rho)$ and (a1) applies in $(0,1)$ if $\delta / \tau=v^{s} \geq 1$; in this case (3.3) holds. If $D$ is given by (3.4) then $D(\rho)=\delta \rho$ and the same result holds true. Therefore, case (a1) applies in $(0,1)$.

Under the Kladek law $(3.12)_{1}$, we pointed out in Sect. 3 that $f$ is strictly concave; if $D$ is as in (3.4), then $D(0)=0$ and $D(\rho)>0$ if $\rho \in(0,1]$. Case (a1) applies in $(0,1)$.

Consider $v(\rho)=\min \{1,-c \log \rho\}$ for $c>0$, see [32]; then the function $f$ is strictly concave in $I=$ $\left(e^{-1 / c}, 1\right)$. If $D$ is given by $(3.1)$, then $D(\rho)=c(\delta-\tau c)$ in $I$ and $D \gtrless 0$ in $I$ if $\delta / \tau \gtrless c$; condition $(3.3)$ holds if $\delta / \tau=v^{s} \geq 1$. Case (a1) ((b1), respectively) applies in $I$.

\section{Proofs}

Proof of Lemma 2.1. Assume that (1.1) has a wavefront solution with profile $\varphi$ satisfying (1.5). We already noticed that $\varphi$ satisfies (2.3) with no loss of generality. Let $\bar{\xi} \geq 0$ be such that $\varphi(\bar{\xi})=\alpha$ and $\varphi(\xi)>\alpha$ if $\xi>\bar{\xi}$. First, we prove 


$$
\text { (i) } \quad \lim _{\xi \rightarrow 0^{-}} D(\varphi(\xi)) \varphi^{\prime}(\xi)=0, \quad \text { (ii) } \quad \lim _{\xi \rightarrow \bar{\xi}^{+}} D(\varphi(\xi)) \varphi^{\prime}(\xi)=0 .
$$

The reasoning is slightly different according to $\bar{\xi}>0$ or $\bar{\xi}=0$; we begin with the case $\bar{\xi}>0$.

Consider $h>0$ such that $h<\xi_{1}$ and $\varphi(\xi)>0$ for $\xi \in(-h, h)$; this is possible because $\alpha \in(0,1)$. For brevity, we denote $F(\xi)=D(\varphi(\xi)) \varphi^{\prime}(\xi)-f(\varphi(\xi))+c \varphi(\xi)$. Let $\psi \in C_{0}^{\infty}(-h, h)$. Since $\varphi$ is a solution to (1.4) (see Definition 2.1), we obtain

$$
0=\int_{-h}^{h} F(\xi) \psi^{\prime}(\xi) \mathrm{d} \xi=\lim _{\varepsilon \rightarrow 0^{+}} \int_{-h}^{-\varepsilon} F(\xi) \psi^{\prime}(\xi) \mathrm{d} \xi+(f(\alpha)-c \alpha) \psi(0)
$$

The function $D(\varphi) \varphi^{\prime}$ is continuous in every interval $(-h,-\varepsilon)$ with $0<\varepsilon<h$, and $\varphi$ satisfies there Eq. (1.4) (see Remark 2.1); hence,

$$
0=\lim _{\varepsilon \rightarrow 0^{+}} \int_{-h}^{-\varepsilon} F(\xi) \psi^{\prime}(\xi) \mathrm{d} \xi+(f(\alpha)-c \alpha) \psi(0)=\lim _{\varepsilon \rightarrow 0^{+}} F(-\varepsilon) \psi(-\varepsilon)+(f(\alpha)-c \alpha) \psi(0) .
$$

Since we may assume $\psi(0) \neq 0$, by the continuity of $f$ and $\varphi$, we obtain condition (5.1) (i). Similarly, one can prove (5.1) (ii) when $\bar{\xi}>0$.

Now, we consider the case $\bar{\xi}=0$. Choose $h>0$ such that $0<\varphi(\xi)<1$ for $\xi \in(-h, h)$ and take $\psi \in C_{0}^{\infty}(-h, h)$. Again by Definition 2.1, we obtain

$$
0=\int_{-h}^{h} F(\xi) \psi^{\prime}(\xi) \mathrm{d} \xi=\lim _{\varepsilon \rightarrow 0^{+}} \int_{-h}^{-\varepsilon} F(\xi) \psi^{\prime}(\xi) \mathrm{d} \xi+\lim _{\varepsilon \rightarrow 0^{+}} \int_{\varepsilon}^{h} F(\xi) \psi^{\prime}(\xi) \mathrm{d} \xi .
$$

For $0<\varepsilon<h$, by the regularity of $\varphi$ in both $(-h,-\varepsilon)$ and $(\varepsilon, h)$ (see Remark 2.1) we obtain

$$
\begin{aligned}
0 & =\lim _{\varepsilon \rightarrow 0^{+}} \int_{-h}^{-\varepsilon} F(\xi) \psi^{\prime}(\xi) \mathrm{d} \xi+\lim _{\varepsilon \rightarrow 0^{+}} \int_{\varepsilon}^{h} F(\xi) \psi^{\prime}(\xi) \mathrm{d} \xi=\lim _{\varepsilon \rightarrow 0^{+}} F(-\varepsilon) \psi(-\varepsilon)-\lim _{\varepsilon \rightarrow 0^{+}} F(\varepsilon) \psi(\varepsilon) \\
& =\lim _{\varepsilon \rightarrow 0^{+}}\left(D(\varphi(-\varepsilon)) \varphi^{\prime}(-\varepsilon)\right) \psi(-\varepsilon)-\lim _{\varepsilon \rightarrow 0^{+}}\left(D(\varphi(\varepsilon)) \varphi^{\prime}(\varepsilon)\right) \psi(\varepsilon) .
\end{aligned}
$$

The above expression must be satisfied in particular when $\psi(0) \neq 0$. Notice, moreover, that $\varphi^{\prime} \geq 0$ in $(-h, h) \backslash\{0\}$ and $D$ changes sign in $\alpha$; hence, both (5.1)(i) and (5.1)(ii) are satisfied. This completely proves (5.1).

Let $\varphi$ be as in the first part of this proof and take $\xi_{1}>0$; the case $\xi_{1}=0$ is proved analogously. We claim that the function

$$
\varphi_{1}(\xi)= \begin{cases}\varphi(\xi) & \text { if } \xi \leq 0, \\ \alpha & \text { if } \xi \in\left(0, \xi_{1}\right), \\ \varphi\left(\xi+\bar{\xi}-\xi_{1}\right) & \text { if } \xi \geq \xi_{1}\end{cases}
$$

is the profile of a wavefront to (1.1). As above, fix $h \in\left(0, \xi_{1}\right)$ such that $\varphi(\xi)>0$ for $\xi \in(-h, 0)$ and consider $\psi \in C_{0}^{\infty}(-h, h)$. By condition (5.1)(i), we obtain

$$
\begin{aligned}
& \int_{-h}^{h}\left(D\left(\varphi_{1}(\xi)\right) \varphi_{1}^{\prime}(\xi)-f\left(\varphi_{1}(\xi)\right)+c \varphi_{1}(\xi)\right) \psi^{\prime}(\xi) \mathrm{d} \xi=\int_{-h}^{0} F(\xi) \psi(0)+(f(\alpha)-c \alpha) \psi(0) \\
= & \lim _{\varepsilon \rightarrow 0^{+}} \int_{-h}^{-\varepsilon} F(\xi) \psi^{\prime}(\xi) \mathrm{d} \xi+(f(\alpha)-c \alpha) \psi(0)=\lim _{\varepsilon \rightarrow 0^{+}} F(-\varepsilon) \psi(-\varepsilon)+(f(\alpha)-c \alpha) \psi(0)=0 .
\end{aligned}
$$


Similarly, by condition (5.1)(ii) we obtain

$$
\int_{\xi_{1}-h}^{\xi_{1}+h}\left(D\left(\varphi_{1}(\xi)\right) \varphi_{1}^{\prime}(\xi)-f\left(\varphi_{1}(\xi)\right)+c \varphi_{1}(\xi)\right) \psi^{\prime}(\xi) \mathrm{d} \xi=0,
$$

for $h \in\left(0, \xi_{1}\right)$ with $\varphi(\bar{\xi}+h)<1$ and $\psi \in C_{0}^{\infty}\left(\xi_{1}-h, \xi_{1}+h\right)$. Hence, $\varphi_{1}$ is the profile of a wavefront of (1.1) with $\varphi_{1}( \pm \infty)=\ell^{ \pm}$.

Proof of Theorem 2.1. Assume that there exists a profile $\varphi$ satisfying (1.5) with speed $c$; by Lemma 2.1 we may assume that it satisfies (2.5). We have to show that $c=c_{\ell^{ \pm}}$, i.e., $c$ satisfies (2.6), and that conditions (2.7), (2.8) hold true. Consider

$$
\varphi_{1}(\xi)=\left\{\begin{aligned}
\varphi(\xi) & \text { if } \xi \leq 0 \\
\alpha & \text { if } \xi>0
\end{aligned}\right.
$$

As in the proof of Lemma 2.1, by condition (5.1)(i) we deduce that $\varphi_{1}$ is the profile of a wavefront to (1.1). By Theorem 4.1(a1), the speed of $\varphi_{1}$ is $c_{1}:=\left[f(\alpha)-f\left(\ell^{-}\right)\right] /\left(\alpha-\ell^{-}\right)$, and condition $(2.7)_{1}$ is satisfied; then, $c=c_{1}$. Moreover, by Theorem 4.1(ii) we deduce $D /(f-s) \in L^{1}\left(I_{\alpha}^{-}\right)$, where $I_{\alpha}^{-}$is a left neighborhood of $\alpha$. Similarly, the function

$$
\varphi_{2}(\xi)=\left\{\begin{aligned}
\alpha & \text { if } \xi<0 \\
\varphi(\xi) & \text { if } \xi \geq 0
\end{aligned}\right.
$$

is the profile of a wavefront of (1.1) with $\varphi_{2}(-\infty)=\alpha, \varphi_{2}(\infty)=\ell^{+}$; by Theorem 4.1(b2), its speed is $c_{2}:=\left[f\left(\ell^{+}\right)-f(\alpha)\right] /\left(\ell^{+}-\alpha\right)$ and condition $(2.7)_{2}$ is satisfied; moreover, $c=c_{2}$. By Theorem 4.1(i) we deduce $D /(f-s) \in L^{1}\left(I_{\alpha}^{+}\right)$, where $I_{\alpha}^{+}$is a right neighborhood of $\alpha$. We deduce $c=c_{1}=c_{2}$ and so (2.6) is satisfied. So (2.6), (2.7) are satisfied if $\varphi$ exists.

Conversely, assume (2.6)-(2.8). Equation (1.1) with $\rho \in\left[\ell^{-}, \alpha\right]$ satisfies assumption (a1) of Theorem 4.1. Hence, it has a wavefront solution with profile $\varphi^{-}$from $\ell^{-}$to $\alpha$, speed $c_{\ell^{ \pm}}$and $\left(\varphi^{-}\right)^{\prime}(\xi)>0$ for $\ell^{-}<\varphi^{-}(\xi)<\alpha$. Since $D /\left(f-s_{\ell^{ \pm}}\right) \in L^{1}\left(I_{\alpha}^{-}\right)$for some left neighborhood $I_{\alpha}^{-}$of $\alpha$ by $(2.8)$, there exists $\xi_{0} \in \mathbb{R}$ such that $\varphi^{-}\left(\xi_{0}\right)=\alpha$ and $\varphi^{-}(\xi)<\alpha$ for $\xi<\xi_{0}$ by Theorem 4.1(ii); we may assume $\xi_{0}=0$. By Theorem 4.1(ii), we deduce

$$
\lim _{\xi \rightarrow 0^{-}} D\left(\varphi^{-}(\xi)\right)\left(\varphi^{-}\right)^{\prime}(\xi)=0 .
$$

Equation (1.1) with $\rho \in\left[\alpha, \ell^{+}\right]$satisfies assumption (b2) of Theorem 4.1; therefore, it has a wavefront solution with profile $\varphi^{+}$from $\alpha$ to $\ell^{+}$, speed $c_{\ell^{ \pm}}$and $\left(\varphi^{+}\right)^{\prime}(\xi)>0$ for $\alpha<\varphi^{+}(\xi)$. Since $D /\left(f-s_{\ell^{ \pm}}\right) \in$ $L^{1}\left(I_{\alpha}^{+}\right)$for some right neighborhood $I_{\alpha}^{+}$of $\alpha$ by (2.8), as above we can assume $\varphi^{+}(0)=\alpha$ with $\varphi^{+}(\xi)>\alpha$ for $\xi>0$. Again by Theorem 4.1(i), we have

$$
\lim _{\xi \rightarrow 0^{+}} D\left(\varphi^{+}(\xi)\right)\left(\varphi^{+}\right)^{\prime}(\xi)=0 .
$$

We now consider the function $\varphi$ defined by $\varphi(\xi)=\varphi^{-}(\xi)$ if $\xi \in(-\infty, 0]$ and $\varphi(\xi)=\varphi^{+}(\xi)$ if $\xi \in(0,+\infty)$. By the properties of $\varphi^{ \pm}$and (5.2)-(5.3), we have that $\varphi$ is a solution to (1.4) in $\mathbb{R}$ (see Definition 2.1 and the reasoning in the first part of this proof) with same $c=c_{\ell^{ \pm}}$. Hence, $\varphi$ is the profile of a wavefront of (1.1) and satisfies all the required properties.

By (2.7), we have $(f(\rho)-f(\alpha)) /(\rho-\alpha)<\left(f(\alpha)-f\left(\ell^{-}\right)\right) /\left(\alpha-\ell^{-}\right)$, for $\rho \in\left(\ell^{-}, \alpha\right)$. Then, we obtain (2.9) by passing to the limit when $\rho \rightarrow \alpha^{-}$.

At last, we prove (2.10). Let $\xi_{\ell^{-}}:=\inf \left\{\xi \in \mathbb{R}: \varphi(\xi)>\ell^{-}\right\}$. Then $\xi_{\ell^{-}} \in \mathbb{R}$ iff $\ell^{-}=0=D(0)$ and $D /\left(f-s_{\ell^{ \pm}}\right) \in L^{1}\left(I_{\ell^{-}}^{+}\right)$, for some right neighborhood $I_{\ell^{-}}^{+}$of $\ell^{-}$, while $\xi_{\ell^{-}}=-\infty$ otherwise. Since $\varphi$ coincides with $\varphi^{-}$when $\xi<0$, by Theorem 4.1(i), (iii) we obtain

$$
\lim _{\xi \rightarrow \xi_{\ell^{-}}} D(\varphi(\xi)) \varphi^{\prime}(\xi)=0 .
$$


By integrating Eq. (1.4) in an interval $\left[\xi_{0}, \xi\right] \subset\left(\xi_{\ell^{-}}, 0\right)$, we have, by Remark 2.1,

$$
D(\varphi(\xi)) \varphi^{\prime}(\xi)-D\left(\varphi\left(\xi_{0}\right)\right) \varphi^{\prime}\left(\xi_{0}\right)+c_{\ell^{ \pm}}\left(\varphi(\xi)-\varphi\left(\xi_{0}\right)\right)-f(\varphi(\xi))+f\left(\varphi\left(\xi_{0}\right)\right)=0 .
$$

Hence, by passing to the limit when $\xi_{0} \rightarrow \xi_{\ell^{-}}$, by (5.4) we obtain

$$
\varphi^{\prime}(\xi)=\frac{f(\varphi(\xi))-f\left(\ell^{-}\right)-c_{\ell^{ \pm}}\left(\varphi(\xi)-\ell^{-}\right)}{D(\varphi(\xi))}, \quad \xi \in\left(\xi_{\ell^{-}}, 0\right) .
$$

In particular, this shows that $\varphi^{\prime}(\xi)>0$ if $\xi \in\left(\xi_{\ell^{-}}, 0\right)$. By de L'Hospital rule, we obtain

$$
\lim _{\xi \rightarrow 0^{-}} \varphi^{\prime}(\xi)=\lim _{\sigma \rightarrow \alpha^{-}} \frac{f(\sigma)-f\left(\ell^{-}\right)-c_{\ell^{ \pm}}\left(\sigma-\ell^{-}\right)}{D(\sigma)}=\lim _{\sigma \rightarrow \alpha^{-}} \frac{f^{\prime}(\sigma)-c_{\ell^{ \pm}}}{D^{\prime}(\sigma)}
$$

whence $\varphi_{-}^{\prime}(0)$ satisfies $(2.10)$. Since the reasoning for $\varphi_{+}^{\prime}(0)$ is similar, this completely proves $(2.10)$. Notice that by (5.6) we immediately deduce (2.11). The proof is complete.

A byproduct of the proof of Lemma 2.1 is formula (5.5); for profiles $\varphi_{\varepsilon}$ of traveling-wave solutions to (2.17), it can be written as

$$
\varphi_{\varepsilon}^{\prime}(\xi)=\frac{f\left(\varphi_{\varepsilon}(\xi)\right)-f\left(\ell^{-}\right)-c_{\ell^{ \pm}}\left(\varphi_{\varepsilon}(\xi)-\ell^{-}\right)}{\varepsilon D\left(\varphi_{\varepsilon}(\xi)\right)},
$$

for every $\xi$ such that $0<\varphi_{\varepsilon}(\xi)<1$ and $\varphi_{\varepsilon}(\xi) \neq \alpha$.

Lemma 5.1. Under the assumptions of Theorem 2.3 we have

$$
\varphi_{\varepsilon_{1}}(\xi) \leq \varphi_{\varepsilon_{2}}(\xi), \quad \text { for every } \quad \xi<0, \varepsilon_{1}<\varepsilon_{2} .
$$

We have $\varphi_{\varepsilon_{1}}\left(\xi_{0}\right)=\varphi_{\varepsilon_{2}}\left(\xi_{0}\right)$ for some $\xi_{0}<0$ iff $\varphi_{\varepsilon_{1}}(\xi)=\varphi_{\varepsilon_{2}}(\xi)=0$ for every $\xi<\xi_{0}$.

Proof. By (2.9), we know that $f^{\prime}(\alpha) \leq c_{\ell^{ \pm}}$.

(i) Assume $f^{\prime}(\alpha)<c_{\ell^{ \pm}}$. First, we show that (5.8) holds in a left neighborhood of 0 and, then, in the whole interval $(-\infty, 0]$.

(a) By (2.10), we have

$$
\varphi_{\varepsilon_{1}}^{\prime}(0)=\frac{f^{\prime}(\alpha)-c_{\ell^{ \pm}}}{\varepsilon_{1} D^{\prime}(\alpha)}>\frac{f^{\prime}(\alpha)-c_{\ell^{ \pm}}}{\varepsilon_{2} D^{\prime}(\alpha)}=\varphi_{\varepsilon_{2}}^{\prime}(0) .
$$

Notice that both conditions $D^{\prime}(\alpha)<0$ in $(2.18)$ and $f^{\prime}(\alpha)<c_{\ell^{ \pm}}$are needed to deduce (5.9). By $\varphi_{\varepsilon_{1}}(0)=$ $\varphi_{\varepsilon_{2}}(0)=\alpha$, see $(2.3)$, we have $\varphi_{\varepsilon_{1}}(\xi)<\varphi_{\varepsilon_{2}}(\xi)$ for $\xi \in(\tilde{\xi}, 0)$, for some $\tilde{\xi}<0$.

(b) Assume that there exists $\bar{\xi}<\tilde{\xi}$ such that $\varphi_{\varepsilon_{1}}(\bar{\xi})=\varphi_{\varepsilon_{2}}(\bar{\xi})=: \eta>0$ while

$$
\varphi_{\varepsilon_{1}}(\xi)<\varphi_{\varepsilon_{2}}(\xi) \quad \text { if } \quad \xi \in(\bar{\xi}, 0) .
$$

By (5.5), (2.6), $(2.7)_{1}, D(\eta)>0$ and $\varepsilon_{1}<\varepsilon_{2}$ we deduce

$$
\varphi_{\varepsilon_{1}}^{\prime}(\bar{\xi})=\frac{f(\eta)-\left(f(\alpha)+c_{\ell^{ \pm}}(\eta-\alpha)\right.}{\varepsilon_{1} D(\eta)}>\frac{f(\eta)-\left(f(\alpha)+c_{\ell^{ \pm}}(\eta-\alpha)\right.}{\varepsilon_{2} D(\eta)}=\varphi_{\varepsilon_{2}}^{\prime}(\bar{\xi}),
$$

in contradiction with (5.10). This proves claim (5.8) if $f^{\prime}(\alpha)<c_{\ell^{ \pm}}$.

(ii) Assume $f^{\prime}(\alpha)=c_{\ell^{ \pm}}$. The above deduction of (5.9) now fails. We prove (5.8) by approximating $\varphi_{\varepsilon_{1}}$ with a sequence of suitably shifted functions $\varphi_{n}$ of $\varphi_{\varepsilon_{1}}$; a further shift $\varphi_{0}$ of $\varphi_{\varepsilon_{1}}$ is introduced to have a uniform bound from below. As in case (i), the first items below concern the proof of (5.8) in a left neighborhood of 0 . We refer to Fig. 7 .

(a) To avoid the possible degeneracy occurring if $D(0)=\ell^{-}=0$, fix $\sigma \in\left(\ell^{-}, \alpha\right)$ and denote $\varphi_{0}(\xi):=$ $\varphi_{\varepsilon_{1}}\left(\xi+\xi_{0}\right)$, where $\xi_{0}<0$ is such that $\varphi_{0}(0)=\sigma$. Then, fix $\mu \in\left(\ell^{-}, \sigma\right)$ and let $\tau_{0}<0$ satisfy $\varphi_{0}\left(\tau_{0}\right)=\mu$. Both values $\xi_{0}$ and $\tau_{0}$ exist by Theorem 2.1, and $\varphi_{0}^{\prime}(\xi)>0$ for $\xi \in\left[\tau_{0}, 0\right]$. By $\sigma=\varphi_{0}(0)<\varphi_{\varepsilon_{2}}(0)=\alpha$, see (2.3), and arguing as in (i)(b), we deduce

$$
\varphi_{0}(\xi)<\varphi_{\varepsilon_{2}}(\xi) \quad \text { for } \xi \in\left[\tau_{0}, 0\right] .
$$




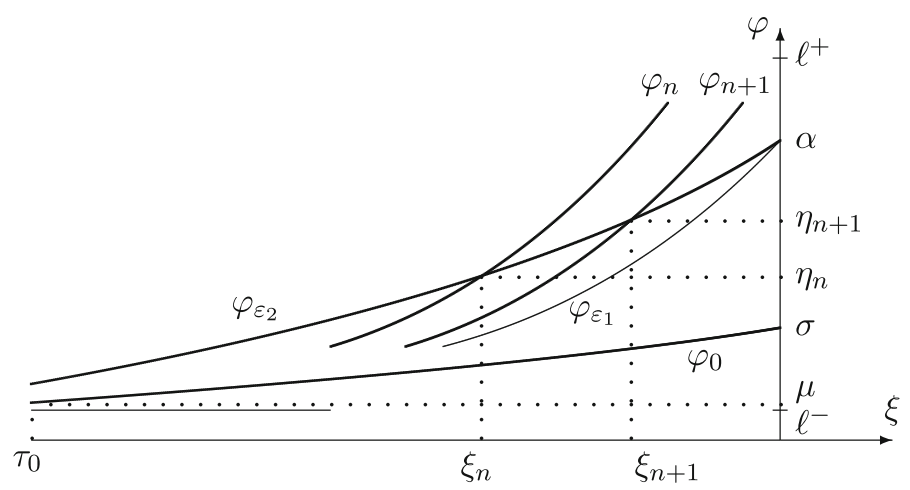

FIG. 7. For the proof of Lemma 5.1

(b) Consider a strictly increasing sequence $\left\{\xi_{n}\right\} \subset\left(\tau_{0}, 0\right)$ satisfying $\xi_{n} \rightarrow 0$ for $n \rightarrow \infty$ and let $\eta_{n}:=\varphi_{\varepsilon_{2}}\left(\xi_{n}\right), n \in \mathbb{N}$. Also the sequence $\left\{\eta_{n}\right\} \subset\left(\ell^{-}, \alpha\right)$ is strictly increasing, by Theorem 2.1. For $n \in \mathbb{N}$, let $\varphi_{n}$ be the solution to the initial-value problem

$$
\left\{\begin{array}{l}
\varphi^{\prime}(\xi)=\frac{f(\varphi(\xi))-f\left(\ell^{-}\right)-c_{\ell \pm}\left(\varphi(\xi)-\ell^{-}\right)}{\varepsilon_{1} D(\varphi(\xi))}, \\
\varphi\left(\xi_{n}\right)=\eta_{n} .
\end{array}\right.
$$

Clearly, we have $\varphi_{n}(\xi)=\varphi_{\varepsilon_{1}}\left(\xi+\zeta_{n}\right)$, for suitable shifts $\zeta_{n}$. As in (ii)(a), we have that $\varphi_{n}$ is defined and strictly increasing in an interval $\left[\tau_{n}, 0\right]$, with $\varphi_{n}\left(\tau_{n}\right)=\mu$. Notice that $\varphi_{0}\left(\xi_{n}\right)<\varphi_{n}\left(\xi_{n}\right)=\eta_{n}$ for every $n$ by (5.11); by the uniqueness of the solution to the equation in (5.12), we deduce $\tau_{n}<\tau_{0}$ and

$$
\varphi_{0}(\xi)<\varphi_{n}(\xi) \quad \text { for } \xi \in\left[\tau_{0}, 0\right] \text { and } n \in \mathbb{N} .
$$

(c) We claim that

$$
\varphi_{n}(\xi)<\varphi_{\varepsilon_{2}}(\xi) \quad \text { for } \xi \in\left[\tau_{0}, \xi_{n}\right) \text { and } n \in \mathbb{N} \text {. }
$$

In fact, as in case $(i)(a)$ with $\xi_{n}$ replacing $\bar{\xi}$, by $(2.7)_{1},(5.7)$ and $D\left(\eta_{n}\right)>0$ we obtain

$$
0<\varphi_{\varepsilon_{2}}^{\prime}\left(\xi_{n}\right)=\frac{f\left(\eta_{n}\right)-f\left(\ell^{-}\right)-c_{\ell^{ \pm}}\left(\eta_{n}-\ell^{-}\right)}{\varepsilon_{2} D\left(\eta_{n}\right)}<\frac{f\left(\eta_{n}\right)-f\left(\ell^{-}\right)-c_{\ell^{ \pm}}\left(\eta_{n}-\ell^{-}\right)}{\varepsilon_{1} D\left(\eta_{n}\right)}=\varphi_{n}^{\prime}\left(\xi_{n}\right) .
$$

Then, $\varphi_{n}(\xi)<\varphi_{\varepsilon_{2}}(\xi)$ in a left neighborhood of $\xi_{n}$; (5.14) follows by arguing as in case (i)(b).

(d) We denote

$$
\hat{\varphi}_{n}(\xi)=\left\{\begin{array}{c}
\varphi_{n}(\xi) \text { if } \tau_{0}<\xi \leq \xi_{n}, \\
\varphi_{\varepsilon_{2}}(\xi) \text { if } \xi_{n}<\xi \leq 0,
\end{array} \quad \text { and } \quad \hat{\varphi}(\xi)=\inf _{n \in \mathbb{N}} \hat{\varphi}_{n}(\xi), \quad \text { for } \xi \in\left[\tau_{0}, 0\right] .\right.
$$

We claim that the sequence $\left\{\hat{\varphi}_{n}\right\}$ is decreasing in $\left[\tau_{0}, 0\right]$. In the interval $\left[\xi_{n+1}, 0\right]$, we have $\varphi_{n+1}=\varphi_{n}=\varphi_{\varepsilon_{2}}$. In $\left(\xi_{n}, \xi_{n+1}\right)$ we have $\varphi_{n+1}<\varphi_{n}=\varphi_{\varepsilon_{2}}$ by (5.14). In $\left[\tau_{0}, \xi_{n}\right)$, we still have $\varphi_{n+1}<\varphi_{n}$ because both of them are shifts of the same profile and are strictly increasing in $\left[\tau_{0}, 0\right]$ by (ii)(b). Then, $\left\{\hat{\varphi}_{n}\right\}$ is decreasing in $\left[\tau_{0}, 0\right]$ and by $(5.11),(5.13)$ we have $\varphi_{0}(\xi) \leq \hat{\varphi}(\xi)=\lim _{n \rightarrow \infty} \hat{\varphi}_{n}(\xi)$ for $\xi \in\left[\tau_{0}, 0\right]$.

(e) Now we prove

$$
\hat{\varphi}=\varphi_{\varepsilon_{1}} \quad \text { in }\left[\tau_{0}, 0\right] .
$$

Equation (5.15) is obviously satisfied in $\xi=0$. Notice that by (2.6) and $(2.7)_{1}$ we have

$$
\psi(\rho):=\frac{f(\rho)-f\left(\ell^{-}\right)-c_{\ell^{ \pm}}\left(\rho-\ell^{-}\right)}{\varepsilon_{1} D(\rho)}>0 \quad \text { for } \rho \in\left(\ell^{-}, \alpha\right) .
$$


Consider an interval $[a, b] \subset\left[\tau_{0}, 0\right)$; since $\psi \in C^{1}([a, b])$, we deduce that $\left\{\hat{\varphi}_{n}\right\}$ is equicontinuous and then relatively compact in $[a, b]$ by Ascoli-Arzelà Theorem. We can extract a subsequence, labeled as the whole sequence, such that $\hat{\varphi}_{n} \rightarrow \hat{\varphi}$ uniformly in $[a, b]$. This implies that also the sequence

$$
\left\{\frac{f\left(\hat{\varphi}_{n}(\xi)\right)-f\left(\ell^{-}\right)-c_{\ell^{ \pm}}\left(\hat{\varphi}_{n}(\xi)-\ell^{-}\right)}{\varepsilon_{1} D\left(\hat{\varphi}_{n}(\xi)\right)}\right\}
$$

is uniformly convergent in $[a, b]$. Choose $N$ large enough in such a way that $\xi_{n}>b$ for $n \geq N$; then $\hat{\varphi}_{n}=\varphi_{n}$ in $[a, b]$ for $n \geq N$. By passing to the limit in the identity

$$
\hat{\varphi}_{n}(b)-\hat{\varphi}_{n}(a)=\int_{a}^{b} \frac{f\left(\hat{\varphi}_{n}(\xi)\right)-f\left(\ell^{-}\right)-c_{\ell^{ \pm}}\left(\hat{\varphi}_{n}(\xi)-\ell^{-}\right)}{\varepsilon_{1} D\left(\hat{\varphi}_{n}(\xi)\right)} \mathrm{d} \xi, \quad n \geq N
$$

we obtain

$$
\hat{\varphi}(b)-\hat{\varphi}(a)=\int_{a}^{b} \frac{f(\hat{\varphi}(\xi))-f\left(\ell^{-}\right)-c_{\ell^{ \pm}}\left(\hat{\varphi}\left(\xi-\ell^{-}\right)\right.}{\varepsilon_{1} D(\hat{\varphi}(\xi))} \mathrm{d} \xi .
$$

This proves (5.15).

(f) By (5.14), condition (5.8) is satisfied in $\left[\tau_{0}, 0\right]$; we prove (5.8) for $\xi<\tau_{0}$ with a reasoning as in (i) (b). This concludes the proof of (5.8).

We are left with the last claim of the statement in the lemma. If either $D(0)>0$ or $\ell^{-}>0$, an argument as in (i)(b) shows that (5.8) is satisfied with the strict inequality. When $D(0)=\ell^{-}=0$, by Theorem 4.1(i) there exists $\xi_{0}<0$ such that $\varphi_{\varepsilon_{1}}(\xi)=\varphi_{\varepsilon_{2}}(\xi)=0$ for $\xi \leq \xi_{0}$ iff and $D /\left(f-s_{\ell^{ \pm}}\right) \in L^{1}\left(I_{\ell^{-}}^{+}\right)$ for some right neighborhood $I_{\ell^{-}}^{+}$of $\ell^{-}$.

Remark 5.1. With reference to the proof of Lemma 5.1, by (5.14) we have $\varphi_{\varepsilon_{1}}\left(\xi_{n}+\zeta_{n+1}\right)=\varphi_{n+1}\left(\xi_{n}\right)<$ $\varphi_{\varepsilon_{2}}\left(\xi_{n}\right)=\varphi_{n}\left(\xi_{n}\right)=\varphi_{\varepsilon_{1}}\left(\xi_{n}+\zeta_{n}\right)$ and so $\zeta_{n+1}<\zeta_{n}$. By arguing as to prove (5.14), one shows $\varphi_{n}>\varphi_{\varepsilon_{2}}$ in $\left(\xi_{n}, 0\right]$, whence $\varphi_{\varepsilon_{1}}\left(\zeta_{n}\right)=\varphi_{n}(0)>\varphi_{\varepsilon_{2}}(0)=\alpha$. Then $0<\zeta_{n+1}<\zeta_{n}$.

Proof of Theorem 2.3. By Lemma 5.1, we can define

$$
\varphi_{0}(\xi):=\lim _{\varepsilon \rightarrow 0^{+}} \varphi_{\varepsilon}(\xi), \quad \xi \leq 0 .
$$

We have $\varphi_{0}(\xi) \geq \ell^{-}$for $\xi \in(-\infty, 0]$; moreover, $\varphi_{0}$ is monotone increasing in $(-\infty, 0]$. As a consequence, if $\varphi_{0}(\bar{\xi})>\ell^{-}$for some $\bar{\xi}<0$ then $\varphi_{0}>\ell^{-}$in the interval $[\bar{\xi}, 0]$.

We prove now (2.19). We reason by contradiction and assume the existence of $\xi_{0}<0$ such that $\varphi_{0}\left(\xi_{0}\right)>\ell^{-}$. By what we have noticed just above, we deduce $\varphi_{0}(\xi)>\ell^{-}$for $\xi \in\left[\xi_{0}, 0\right]$. Let $\xi_{1} \in\left(\xi_{0}, 0\right)$. By the monotonicity of each $\varphi_{\varepsilon}, \varepsilon \in[0,1]$, we have

$$
\ell^{-}<\mu_{0}:=\varphi_{0}\left(\xi_{0}\right) \leq \varphi_{0}(\xi)<\varphi_{\varepsilon}(\xi)<\varphi_{1}(\xi) \leq \varphi_{1}\left(\xi_{1}\right)=: \mu_{1}<\alpha,
$$

for $\xi \in\left[\xi_{0}, \xi_{1}\right], \varepsilon \in(0,1]$. As a consequence, for each $\varepsilon \in(0,1]$, condition $(2.7)_{1}$ implies

$$
\min _{\mu_{0} \leq \rho \leq \mu_{1}}\left[f(\rho)-\left(f(\alpha)+c_{\ell^{ \pm}}(\rho-\alpha)\right)\right]=\sigma,
$$

for some $\sigma>0$. On the other hand, by (5.7) we have

$$
\varphi_{\varepsilon}^{\prime}(\xi) \geq \frac{\sigma}{\varepsilon D\left(\varphi_{\varepsilon}(\xi)\right)} \geq \frac{\sigma}{\varepsilon \max _{\mu_{0} \leq \rho \leq \mu_{1}} D(\rho)}, \quad \xi \in\left[\xi_{0}, \xi_{1}\right], \varepsilon \in(0,1],
$$

which contradicts that $\varphi_{\varepsilon}(0)=\alpha$ for every $\varepsilon>0$. At last, by the estimate

$$
\varphi_{\varepsilon}(\xi) \leq \varphi_{\varepsilon}(\delta), \quad \xi \geq \delta, \varepsilon>0
$$

we obtain the uniform convergence in any half line $[\delta, \infty)$, with $\delta>0$; the reasoning is similar in any half line $(-\infty,-\delta)$, again with $\delta>0$. 
Proof of Lemma 3.2. First, assume that $v$ does not decrease. If $v^{\prime}$ changes sign once, then $v$ first decreases and then increases. This contradicts the assumptions $v \geq 0$ in $[0,1)$ and $v(1)=0$. If $v^{\prime}$ changes sign twice, then it vanishes twice in $(0,1)$ and the same must occur for $D$. This contradicts (D1).

We are left with the case when $v$ decreases. We denote $w(\rho)=\delta+\tau \rho v^{\prime}(\rho)$. In order that (D1) holds we need that $w$ changes sign from the positive to the negative at $\alpha$.

If $w(\rho)=k(\alpha-\rho)$ for some $k>0$, then $v(\rho)=\frac{k \alpha-\delta}{\tau} \log \rho+\frac{k}{\tau}(1-\rho)$, with $k \alpha \leq \delta$ in order that $v$ may decrease. Indeed, we need $k \alpha=\delta$ in order that $v$ is bounded and then, by imposing $v(1)=1$, we have $(3.8)_{1}$. This velocity satisfies (fcm) and we deduce $D(\rho)=\frac{\delta^{2}}{\alpha^{2} \tau} \rho(\alpha-\rho)$.

This example has the drawback that $D(1) \neq 0$; if we define for instance $w(\rho)=k(\alpha-\rho)(1-\rho)$, then, choosing again $k \alpha=\delta$ and imposing $v(1)=0$, we deduce $(3.8)_{2}$ with $D(\rho)=\frac{\delta^{2}}{\alpha^{2} \tau} \rho(\alpha-\rho)(1-\rho)(1+\alpha-\rho)$.

About condition (2.7), in the case $(3.8)_{1}$ the function $f$ is concave. In the latter, it is easy to prove that any line through $(\alpha, f(\alpha))$ intersects the graph of $f$ at most at one point different from $\alpha$. At last, it is obvious from (3.8) that condition (3.3) fails in both cases.

Proof of Lemma 3.3. The line $y=\sigma \rho$ meets the graph of $(1-\rho)^{3}$ precisely at one point in the interval $(0,1)$; this defines $\alpha \in(0,1)$ by $(3.11)$ and then $D$ satisfies (D1). Note that $\alpha$ covers the interval $(0,1)$ when $\sigma$ ranges in $(0, \infty)$; in particular we can take $\alpha \in\left(\frac{1}{2}, 1\right)$ for a suitable choice of the parameters $\bar{v}, h, \tau$.

The pairs $\left(\ell^{-}, \ell^{+}\right)$are constructed by a geometric argument. Denote by $r_{m}(\rho)=m(\rho-\alpha)+f(\alpha)$ a generic line through $(\alpha, f(\alpha))$, parametrized by $m$; we need that the equation $f(\rho)=r_{m}(\rho)$ has two solutions $\rho_{ \pm}$with

$$
0<\rho_{-}<\alpha<\rho_{+}<1 .
$$

We have $f(\rho)=r_{m}(\rho)$ if and only if $\bar{v} \rho(1-\rho)^{2}=m(\rho-\alpha)+\bar{v} \alpha(1-\alpha)^{2}$. Since $\rho(1-\rho)^{2}-\alpha(1-\alpha)^{2}=$ $(\rho-\alpha)\left(\rho^{2}-(2-\alpha) \rho+(1-\alpha)^{2}\right)$, then the previous equation is satisfied iff

$$
\rho^{2}-(2-\alpha) \rho+(1-\alpha)^{2}-\mu=0, \quad \mu:=m / \bar{v} .
$$

We need that (5.18) has roots $\rho_{ \pm}$satisfying (5.17). The discriminant of (5.18) is positive iff

$$
\mu>-\alpha\left(1-\frac{3}{4} \alpha\right) .
$$

If (5.19) holds then the roots of (5.18) are $\rho_{ \pm}=\frac{2-\alpha \pm \sqrt{-3 \alpha^{2}+4 \alpha+4 \mu}}{2}$. Now, we check (5.17).

- We have $0<\rho_{-}$iff $2-\alpha>\sqrt{-3 \alpha^{2}+4 \alpha+4 \mu}$, i.e.,

$$
\mu<(1-\alpha)^{2} \text {. }
$$

- We have $\rho_{-}<\alpha$ iff $2-3 \alpha<\sqrt{-3 \alpha^{2}+4 \alpha+4 \mu}$, i.e.,

$$
\text { either } \alpha \in(2 / 3,1) \quad \text { or } \quad \alpha \in(0,2 / 3] \text { and } \mu>3 \alpha^{2}-4 \alpha+1=(1-\alpha)(1-3 \alpha) \text {. }
$$

- We have $\alpha<\rho_{+}$iff $3 \alpha-2<\sqrt{-3 \alpha^{2}+4 \alpha+4 \mu}$, i.e.,

$$
\text { either } \alpha \in(0,2 / 3) \quad \text { or } \quad \alpha \in[2 / 3,1) \text { and } \mu>3 \alpha^{2}-4 \alpha+1=(1-\alpha)(1-3 \alpha) \text {. }
$$

- We have $\rho_{+}<1$ iff $\sqrt{-3 \alpha^{2}+4 \alpha+4 \mu}<\alpha$, i.e.,

$$
\mu<-\alpha(1-\alpha) \text {. }
$$

Therefore $\mu$ must satisfy conditions (5.19)-(5.23). Clearly, (5.23) implies (5.20); on the other hand, (5.21) and (5.22) imply (5.19) because $3 \alpha^{2}-4 \alpha+1 \geq-\alpha\left(1-\frac{3}{4} \alpha\right)$. Then we require 


$$
(1-\alpha)(1-3 \alpha)<\mu<-\alpha(1-\alpha) .
$$

Notice that $\mu<0$ and the inequality is nonempty iff $\alpha>1 / 2$, whence the requirement in the statement. The pairs $\left(\ell^{-}, \ell^{+}\right)$are found as follows: choose any $m$ in the interval $(\bar{v}(1-\alpha)(1-3 \alpha),-\bar{v} \alpha(1-\alpha))$; then, the abscissas (different from $\alpha$ ) of the points of intersection of the line $r_{m}$ with the graph of $f$ can be taken as $\ell^{ \pm}$. These end states $\ell^{ \pm}$satisfy both conditions (2.6) and (2.7). Now we prove

$$
f^{\prime}(\alpha)<m \quad \text { for } \quad m \in(\bar{v}(1-\alpha)(1-3 \alpha),-\bar{v} \alpha(1-\alpha)),
$$

so that also condition (2.8) is satisfied by comment (ii) to Theorem 2.1. Assume first $\alpha \neq 2 / 3$; then the function $f$ is either strictly concave or strictly convex at point $\alpha$. The line $r_{m}$ cannot be tangent to the graph of $f$ at the point $(\alpha, f(\alpha))$, because by convexity the graph would stay either below $r_{m}$ for $\rho<\alpha$ when $\alpha<2 / 3$ or above $r_{m}$ for $\rho>\alpha$ if $\alpha>2 / 3$, thus contradicting (2.7). Then, $f^{\prime}(\alpha)<m$. If $\alpha=2 / 3$, i.e., if $\alpha$ is the inflection point of $f$, then $f^{\prime}(2 / 3)=-\bar{v} / 3$; the allowed range for $m$ is $(-\bar{v} / 3,-2 \bar{v} / 9)$. So we obtain again $f^{\prime}(\alpha)<m$.

Proof of Lemma 3.4. We have $v^{\prime}(\rho)=-\rho^{2}+(\alpha+\beta) \rho-\alpha \beta$. By integration and imposing the condition $v(1)=0$, we deduce $v(\rho)=-\rho^{3} / 3+(\alpha+\beta) \rho^{2} / 2-\alpha \beta \rho+\gamma$, where $\gamma:=1 / 3-(\alpha+\beta) / 2+\alpha \beta$. We need to check when $v(\rho) \geq 0$ for $\rho \in[0,1)$. By (3.9), in the interval $[0,1]$ the function $v$ has two minimum points at $\alpha$ and 1 . We need to prove when $v(\alpha) \geq 0$. We have $v(\alpha)=(\alpha-1)^{2}(\alpha-3 \beta+2) / 6$. At last, under (3.16)-(3.17), the velocity $v$ is decreasing in $[0, \alpha) \cup(\beta, 1]$ and increasing in $(\alpha, \beta)$. Hence, $v$ has a minimum at $\alpha$ and $v(\alpha)<v(\beta)$. Then,

$$
\frac{f(\beta)-f(\alpha)}{\beta-\alpha}=\frac{\beta v(\beta)-\alpha v(\alpha)}{\beta-\alpha}>0 .
$$

This proves the lemma.

\section{Acknowledgements}

The authors are members of the Gruppo Nazionale per l'Analisi Matematica, la Probabilità e le loro Applicazioni (GNAMPA) of the Istituto Nazionale di Alta Matematica (INdAM) and acknowledge financial support from this institution.

Publisher's Note Springer Nature remains neutral with regard to jurisdictional claims in published maps and institutional affiliations.

\section{References}

[1] Aw, A., Rascle, M.: Resurrection of "second order" models of traffic flow. SIAM J. Appl. Math. 60(3), 916-938 (2000)

[2] Bao, L., Zhou, Z.: Traveling wave in backward and forward parabolic equations from population dynamics. Discrete Contin. Dyn. Syst. Ser. B 19(6), 1507-1522 (2014)

[3] Bao, L., Zhou, Z.: Traveling wave solutions for a one dimensional model of cell-to-cell adhesion and diffusion with monostable reaction term. Discrete Contin. Dyn. Syst. Ser. S 10(3), 395-412 (2017)

[4] Bellomo, N., Delitala, M., Coscia, V.: On the mathematical theory of vehicular traffic flow. I. Fluid dynamic and kinetic modelling. Math. Models Methods Appl. Sci. 12(12), 1801-1843 (2002)

[5] Bellomo, N., Dogbe, C.: On the modeling of traffic and crowds: a survey of models, speculations, and perspectives. SIAM Rev. 53(3), 409-463 (2011)

[6] Bressan, A.: Hyperbolic Systems of Conservation Laws. Oxford University Press, Oxford (2000)

[7] Bruno, L., Tosin, A., Tricerri, P., Venuti, F.: Non-local first-order modelling of crowd dynamics: a multidimensional framework with applications. Appl. Math. Model. 35(1), 426-445 (2011)

[8] Coclite, G.M., Corli, A., di Ruvo, L.: Vanishing viscosity limits of scalar equations with degenerate diffusivity (2017) 
[9] Colombo, R.M., Rosini, M.D.: Pedestrian flows and non-classical shocks. Math. Methods Appl. Sci. 28(13), 1553-1567 (2005)

[10] Corli, A., di Ruvo, L., Malaguti, L., Rosini, M.D.: Traveling waves for degenerate diffusive equations on networks. Netw. Heterog. Media 12(3), 339-370 (2017)

[11] Dafermos, C.M.: Hyperbolic Conservation Laws in Continuum Physics, 3rd edn. Springer, Berlin (2010)

[12] DiCarlo, D.A., Juanes, R., Tara, L., Witelski, T.P.: Nonmonotonic traveling wave solutions of infiltration into porous media. Water Resour. Res. 44, 1-12 (2008)

[13] Evans, L.C., Portilheiro, M.: Irreversibility and hysteresis for a forward-backward diffusion equation. Math. Models Methods Appl. Sci. 14(11), 1599-1620 (2004)

[14] Ferracuti, L., Marcelli, C., Papalini, F.: Travelling waves in some reaction-diffusion-aggregation models. Adv. Dyn. Syst. Appl. 4(1), 19-33 (2009)

[15] Garavello, M., Han, K., Piccoli, B.: Models for vehicular traffic on networks. American Institute of Mathematical Sciences (AIMS), Springfield, MO (2016)

[16] Gilding, B.H., Kersner, R.: Travelling Waves in Nonlinear Diffusion-convection Reaction. Birkhäuser Verlag, Basel (2004)

[17] Gilding, B.H., Tesei, A.: The Riemann problem for a forward-backward parabolic equation. Phys. D 239(6), 291-311 (2010)

[18] Helbing, D.: Traffic and related self-driven many-particle systems. Rev. Mod. Phys. 73, 1067-1141 (2001)

[19] Herty, M., Illner, R.: On stop-and-go waves in dense traffic. Kinet. Relat. Models 1(3), 437-452 (2008)

[20] Herty, M., Puppo, G., Roncoroni, S., Visconti, G.: Propagation of waves in kinetic traffic models. (2018)

[21] Höllig, K.: Existence of infinitely many solutions for a forward backward heat equation. Trans. Am. Math. Soc. 278(1), 299-316 (1983)

[22] Horstmann, D., Painter, K.J., Othmer, H.G.: Aggregation under local reinforcement: from lattice to continuum. Eur. J. Appl. Math. 15(5), 546-576 (2004)

[23] Kerner, B.S.: Experimental features of self-organization in traffic flow. Phys. Rev. Lett. 81, 3797-3800 (1998)

[24] Kerner, B.S., Osipov, V.V.: Autosolitons. Kluwer Academic Publishers Group, Dordrecht (1994)

[25] Kružkov, S.N.: First order quasilinear equations with several independent variables. Mat. Sb. 81(123), 228-255 (1970)

[26] Kuzmin, M., Ruggerini, S.: Front propagation in diffusion-aggregation models with bi-stable reaction. Discrete Contin. Dyn. Syst. Ser. B 16(3), 819-833 (2011)

[27] LeFloch, P.G.: Hyperbolic systems of conservation laws. Lectures in Mathematics ETH Zürich. Birkhäuser Verlag, Basel (2002)

[28] Lighthill, M .J., Whitham, G .B.: On kinematic waves. II. A theory of traffic flow on long crowded roads. Proc. R. Soc. Lond. Ser. A. 229, 317-345 (1955)

[29] Maini, P.K., Malaguti, L., Marcelli, C., Matucci, S.: Diffusion-aggregation processes with mono-stable reaction terms. Discrete Contin. Dyn. Syst. Ser. B 6(5), 1175-1189 (2006)

[30] Maini, P.K., Malaguti, L., Marcelli, C., Matucci, S.: Aggregative movement and front propagation for bi-stable population models. Math. Models Methods Appl. Sci. 17(9), 1351-1368 (2007)

[31] Mascia, C., Terracina, A., Tesei, A.: Two-phase entropy solutions of a forward-backward parabolic equation. Arch. Ration. Mech. Anal. 194(3), 887-925 (2009)

[32] Nelson, P.: Synchronized traffic flow from a modified Lighthill-Whitham model. Phys. Rev. E 61, R6052-R6055 (2000)

[33] Nelson, P.: Traveling-wave solutions of the diffusively corrected kinematic-wave model. Math. Comput. Model. 35(5-6), 561-579 (2002)

[34] Payne, H.J.: Models of freeway traffic and control. Simul. Counc. Proc. 1, 51-61 (1971)

[35] Plotnikov, P. I.: Passage to the limit with respect to viscosity in an equation with a variable direction of parabolicity. Differentsial' nye Uravneniya, 30(4):665-674, 734 (1994)

[36] Richards, P.I.: Shock waves on the highway. Oper. Res. 4, 42-51 (1956)

[37] Rosini, M.D.: Macroscopic Models for Vehicular Flows and Crowd Dynamics: Theory and Applications. Springer, Heidelberg (2013)

[38] Smarrazzo, F., Tesei, A.: Degenerate regularization of forward-backward parabolic equations: the regularized problem. Arch. Ration. Mech. Anal. 204(1), 85-139 (2012)

[39] Smarrazzo, F., Tesei, A.: Degenerate regularization of forward-backward parabolic equations: the vanishing viscosity limit. Math. Ann. 355(2), 551-584 (2013)

[40] Terracina, A.: Non-uniqueness results for entropy two-phase solutions of forward-backward parabolic problems with unstable phase. J. Math. Anal. Appl. 413(2), 963-975 (2014)

[41] Terracina, A.: Two-phase entropy solutions of forward-backward parabolic problems with unstable phase. Interfaces Free Bound. 17(3), 289-315 (2015)

[42] Venuti, F., Bruno, L.: An interpretative model of the pedestrian fundamental relation. C. R. Mech. 335, 194-200 (2007)

[43] Volpert, A .I., Hudjaev, S .I.: The Cauchy problem for second order quasilinear degenerate parabolic equations. Mat. Sb. (N.S.) 78(120), 374-396 (1969) 
[44] Witelski, T.P.: The structure of internal layers for unstable nonlinear diffusion equations. Stud. Appl. Math. 97(3), 277-300 (1996)

[45] Zhang, H.M.: A non-equilibrium traffic model devoid of gas-like behavior. Transp. Res. B 36, 275-290 (2002)

Andrea Corli

Department of Mathematics and Computer Science

University of Ferrara

44121 Ferrara

Italy

e-mail: andrea.corli@unife.it

Luisa Malaguti

Department of Sciences and Methods for Engineering

University of Modena and Reggio Emilia

42122 Modena

Italy

e-mail: luisa.malaguti@unimore.it

(Received: May 17, 2018; revised: February 8, 2019) 